Supporting Information for:

\title{
Catalytic Performance and Electrophoretic Behavior of an Yttrium-Organic Framework based on a Tricarboxylic Asymmetric Alkyne
}

Juana M. Pérez, ${ }^{[a], 1}$ Sara Rojas, ${ }^{[b], 1}$ Amalia Garcia-Garcia, ${ }^{[b]}$ Helena Montes-Andrés, ${ }^{[b]}$ Cristina Ruiz Martínez, ${ }^{[a]}$ Manuel S. Romero-Cano, ${ }^{[a]}$ Duane Choquesillo-Lazarte, ${ }^{[c]}$ Victor Karim Abdelkader-Fernández, ${ }^{[b]}$ Manuel Pérez-Mendoza, ${ }^{[b]}$ Javier Cepeda, ${ }^{[d]}$ Antonio Rodríguez-Diéguez, *[b] and Ignacio Fernández*[a]

[a] Dr. Juana M. Pérez, Ms. Cristina Ruiz Martínez, Dr. Manuel S. Romero-Cano, Prof. Dr. Ignacio Fernández, Department of Chemistry and Physics, University of Almería, Ctra. Sacramento s/n, 04120 Almería, Spain, E-mail: ifernan@ual.es

[b] Dr. Sara Rojas, Dr. Amalia García-García, Dr. Víctor Karim Abdelkader-Fernández, Prof. Manuel Pérez-Mendoza, Dr. Helena Montes-Andrés, Prof. Dr. Antonio Rodríguez-Diéguez, Department of Inorganic Chemistry, University of Granada, Av. Fuentenueva s/n, 18071 Granada, Spain, E-mail: antonio5@ugr.es

[c] Dr. Duane Choquesillo-Lazarte, Laboratorio de Estudios Cristalográficos, IACT, CSIC-Universidad de Granada, Avda. de las Palmeras 4, 18100 Armilla, Granada, Spain.

[d] Dr. Javier Cepeda, Departamento de Química Aplicada, Universidad del País Vasco (UPV/EHU), Paseo Manuel Lardizabal, 3, 20018, Donostia-San Sebastián, Spain.

1 These authors have contributed equally. 


\section{Table of Contents:}

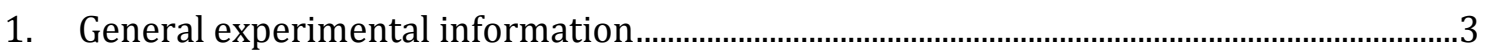

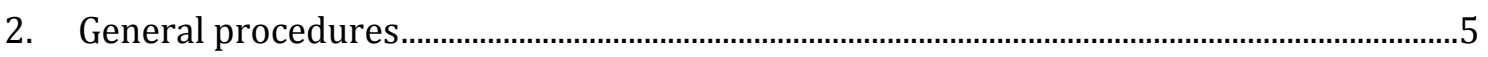

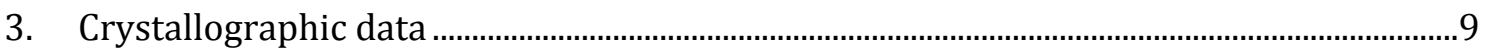

4. Selected bond lengths and angles data …………................................................................

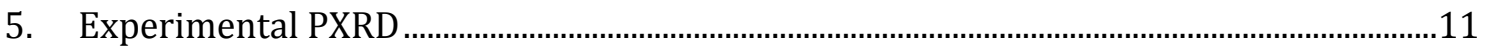

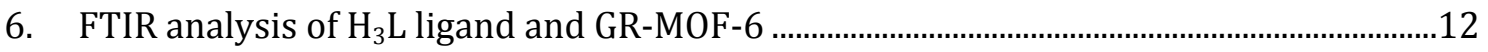

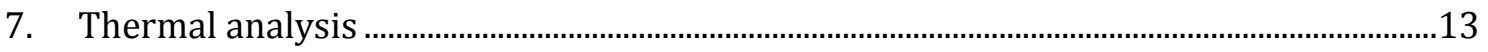

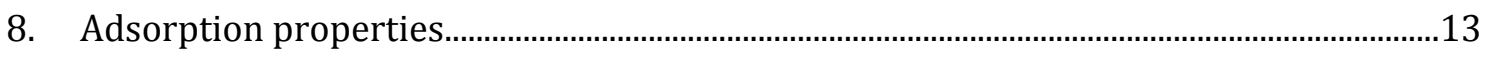

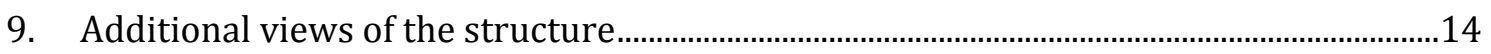

10. Study of $\zeta$-potential at different $\mathrm{pH}$ values and size distribution.......................................15

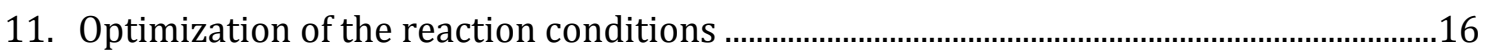

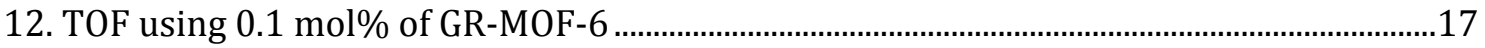

13. Characterization Data of Products.............................................................................................

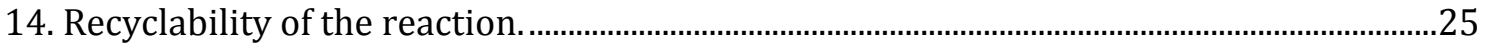

15. Studies of the mechanism of the reaction using IR (ATR).....................................................26

16. Previous results obtained with different lanthanides MOF catalysts ...................................27

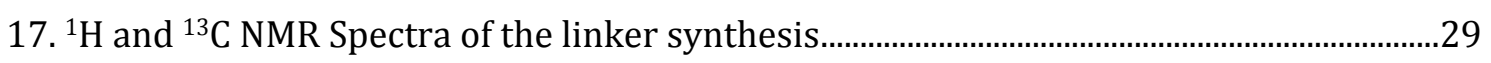

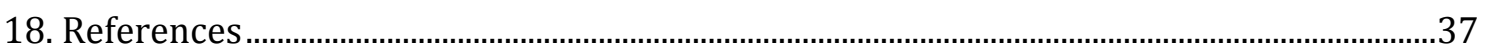




\section{General experimental information}

All experiments involving moisture-sensitive compounds were performed under an inert atmosphere of $\mathrm{N}_{2}$ using standard techniques. Unless otherwise indicated, reagents and substrates were purchased from commercial sources and used as received. Solvents not required to be dry were purchased as technical grade and used as received.

NMR measurements: NMR spectra were measured in a Bruker Avance III 300 spectrometer equipped with a direct double SmartProbe BBFO 1H/BB(19F) probe. Chemical shifts are reported in parts per million (ppm) relative to external TMS for ${ }^{1} \mathrm{H}$ and ${ }^{13} \mathrm{C}$. Coupling constants are reported in Hertz. Multiplicity is reported with the usual abbreviations (s: singlet, bs: broad singlet, d: doublet, dd: doublet of doublets, ddd: doublet of doublet of doublets, t: triplet, td: triplet of doublets, q: quartet, dq: doublet of quartet, p: pentet, sex: sextet, hept: heptet, m: multiplet).

Infrared Attenuated total reflection (IR-ATR) spectra of products were recorded in a FT-IR Bruker Alpha spectrometer. IR spectra of 5-((4-carboxyphenyl)ethynyl) isophthalic acid $\left(\mathrm{H}_{3} \mathrm{~L}\right)$ ligand and compound GRMOF-6 were recorded in the region $400-4000 \mathrm{~cm}^{-1}$ on a Nicolet 6700 FTIR (Fourier transform infrared) spectrophotometer (Thermo Phisher Scientific, TX, USA) with samples as KBr disks.

Elemental analyses (EA) of synthetized new compounds were performed on an Elementar vario EL cube in the $\mathrm{CHN}$ mode.

Gasses $\left(\mathbf{N}_{\mathbf{2}}\right.$ and $\left.\mathbf{C O}_{2}\right)$ sorption experiments were carried out in a Micromeritics ASAP2020 equipment. Prior to the measurements, the sample was first activated at $120^{\circ} \mathrm{C}$ overnight under vacuum $(300 \mathrm{mmHg})$.

Thermogravimetric analysis (TG/DTA) were performed on a TG-Q500 TA Instruments thermal analyser from room temperature to $800{ }^{\circ} \mathrm{C}$ under a synthetic air atmosphere $\left(79 \% \mathrm{~N}_{2} / 21 \% \mathrm{O}_{2}\right)$ at a heating rate of $10{ }^{\circ} \mathrm{C}$ $\min ^{-1}$.

X-ray Diffraction Data Collection and Structure Determination. Single-crystal diffraction data were collected at 100(2) K on a Bruker X8 APEX II and Bruker D8 Venture with a Photon detector equipped with graphite monochromated MoK $\alpha$ radiation $(\lambda=0.71073 \AA)$. The data reduction was performed with the APEX2 software $^{1}$ and corrected for absorption using SADABS. ${ }^{2}$ These structures were solved by direct methods using the SHELXT program ${ }^{3}$ and refined by full-matrix least-squares of $F^{2}$ including all reflections with SHELXL2018/3 program. ${ }^{4}$ All calculations for these structures were performed using the WINGX crystallographic software package. ${ }^{5}$ Final $R(F), w R\left(F^{2}\right)$ and goodness of fit agreement factors, details on the data collection and analysis can be found in Table S1. CCDC 2092074 contain the supplementary crystallographic data for GRMOF-6. These data can be obtained free of charge from The Cambridge Crystallographic Data Centre via www.ccdc.cam.ac.uk/data_request/cif.

Powder X-ray diffraction (XRPD) patterns were collected on a Philips X'PERT powder diffractometer with $\mathrm{Cu} \mathrm{K} \alpha$ radiation $(\lambda=1.5418 \AA)$ over the range of $5<2 \theta<50^{\circ}$ with a step size of $0.02^{\circ}$ and an acquisition time of $2.5 \mathrm{~s}$ per step at $25{ }^{\circ} \mathrm{C}$. Indexation of the diffraction profiles were carried out using the FULLPROF program, ${ }^{6}$ on the basis of the space group and cell parameters found for isostructural compounds by single crystal X-ray diffraction. 
Conversion values relative to the limiting reagent were calculated from the ${ }^{1} \mathrm{H}$ NMR spectra of the reaction crudes. Isolated products were obtained after centrifugation $(8000 \mathrm{rpm}, 3 \mathrm{~min})$ and washed with dichloromethane (DCM, $2 \times 0.5 \mathrm{~mL}$ ) in order to remove the catalyst or column chromatography in silica gel using hexane as eluent.

DLS measurements were performed using the ZetaMaster S ZEM5002 instrument, quartz cells with a path length of $1 \mathrm{~cm}$ and laser wavelength of $670 \mathrm{~nm}$. Electrophoretic mobility and Z-potential measurements were made in a ZetaSizer Nano-Z ZEN2600 instrument, DTS1070 cell type and laser wavelength of $632.8 \mathrm{~nm}$. Samples were prepared at $0.05 \% \mathrm{w} / \mathrm{w}$, dispersing $2.5 \mathrm{mg}$ of the powder and making up to weight with distilled water. Two vials per measuring point were prepared, adjusting the $\mathrm{pH}$ manually under magnetic stirring with $\mathrm{NaOH}$ and $\mathrm{HCl}$ solutions. The dispersions were sonicated after preparation for 1 min to tear the aggregates. Subsequently, they were shaken and left for sedimentation for another $5 \mathrm{~min}$. After this time, the supernatant was extracted, and the deposit was placed in the stove at $70^{\circ} \mathrm{C}$ to estimate the representative amount of sample studied. Afterwards, the $\mathrm{pH}$ of the supernatants was checked, and the conductivity was set to $320 \mu \mathrm{S} / \mathrm{cm}$ with $\mathrm{NaCl} 0.1 \mathrm{M}$. Samples were shaken vigorously prior to their insertion into the measuring devices. Equilibration time was set to $120 \mathrm{~s}$. Average results obtained from 4 measurements for DLS, and 9 for electrophoretic mobility.

Optic micrograph analysis was carried out with a Nikon Eclipse Ti-E motorized inverted microscope equipped with objectives: Plan Fluor 10x, Plan apochromatic 20x, 40x, 60x oil, with a transmission detector. 


\section{General procedures}

General procedure for the synthesis of the organic linker $\left(\mathrm{H}_{3} \mathbf{L}\right)$ : The preparation of the organic linker 5((4-carboxiphenyl)ethynyl) isophthalic acid involves five steps. Amine dimethyl 5-aminoisophthalate (3) was used as starting material. Through its reaction with $\mathrm{NaNO}_{2}$ and $\mathrm{KI}$ in toluene, the iodide derivative 4a was obtained after the purification by recrystallization in $\mathrm{MeOH}$, as a light-yellow solid (65\%). Next, the silicon derivative 5a was obtained through reaction of dimethyl 5-iodoisophthalate $\mathbf{4 a}$ with $\mathrm{CuI}, \mathrm{PPh}_{3}$ and $\mathrm{Pd}\left(\mathrm{PPh}_{3}\right)_{2} \mathrm{Cl}_{2}$ in $\mathrm{Et}_{3} \mathrm{~N}$ as solvent, following by the addition of ethynyltrimethylsilane, with a reaction yield of $80 \%$. This silicon derivative was treated with $\mathrm{KF}$ in $\mathrm{EtOH}$ as solvent for obtaining the alkine 6. Finally, compounds 6 and 7 were used in the Sonogashira reaction, which was catalysed by $\mathrm{Pd}\left(\mathrm{PPh}_{3}\right)_{2} \mathrm{Cl}_{2}$ in toluene as solvent. Compound 8 was purified by column chromatography $\left(\mathrm{SiO}_{2}\right)$, with a yield of $67 \%$, and finally hydrolysed, obtaining the desired ligand $\mathbf{H}_{3} \mathbf{L}$ with an excellent yield (90\%).

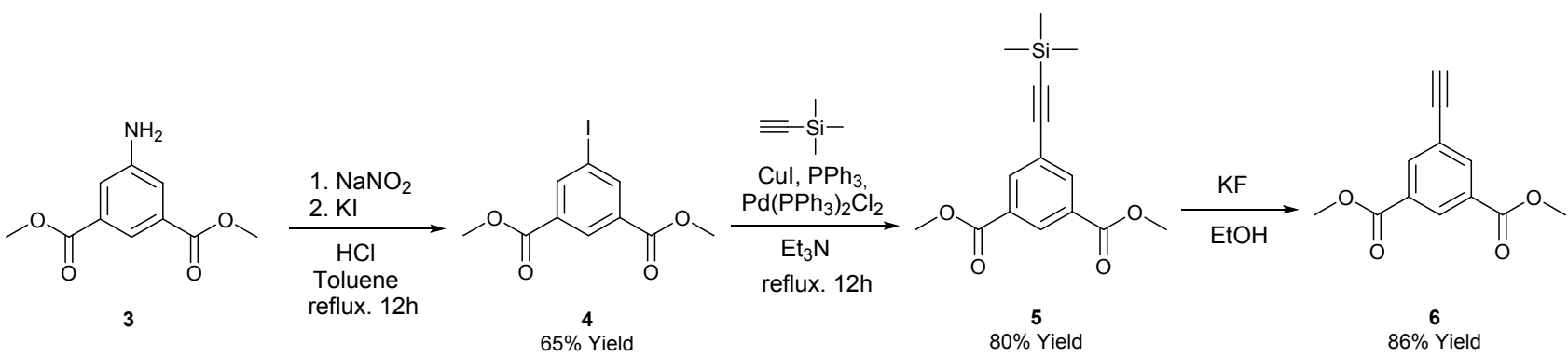

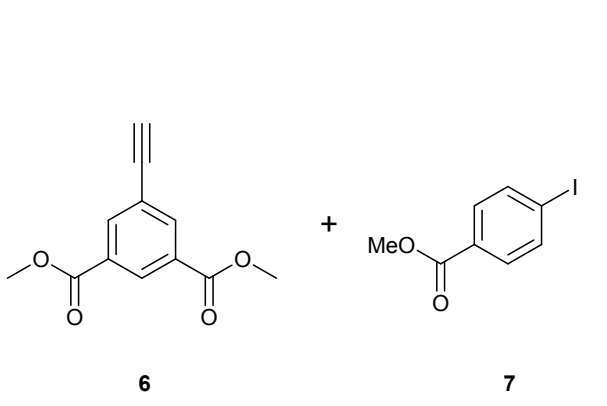
7
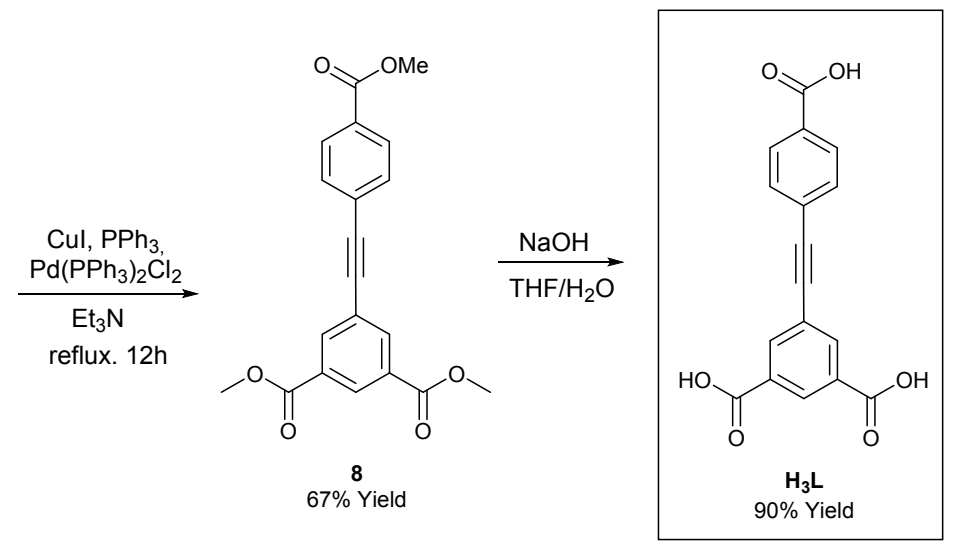

Scheme S1. The full synthetic procedure of $\mathrm{H}_{3} \mathrm{~L}$.

\section{General procedure for the synthesis of dimethyl 5-iodoisophthalate (4):}<smiles>COC(=O)c1cc(I)cc(C(=O)OC)c1</smiles>

After preparing a suspension of dimethyl 5-aminoisophthalate $3(5.2 \mathrm{~g}, 25 \mathrm{mmol})$ in $6 \mathrm{M} \mathrm{HCl}(15 \mathrm{~mL})$ at $0{ }^{\circ} \mathrm{C}$, a solution of sodium nitrite $1.7 \mathrm{M}(30 \mathrm{~mL})$ was added dropwise under stirring. Next, $40 \mathrm{~mL}$ of toluene was added followed by the gradual addition of a solution of $8.2 \mathrm{~g}$ of KI previously dissolved in $20 \mathrm{~mL}$ of $\mathrm{H}_{2} \mathrm{O}$. The reaction mixture was left under stirring for $12 \mathrm{~h}$. The crude of reaction was then extracted three times with ethyl acetate. The collected organic phases were dried over anhydrous $\mathrm{Na}_{2} \mathrm{SO}_{4}$ and the solvent was removed under vacuum. The resulting brown oil was recrystallized from methanol, obtaining compound 4 as a light-yellow solid (yield: $65 \%, 5.2 \mathrm{~g}) .{ }^{1} \mathrm{H} \mathrm{RMN}\left(400 \mathrm{MHz}, \mathrm{CDCl}_{3}\right): \delta=8.61(\mathrm{t}, J=1.4 \mathrm{~Hz}$, $1 \mathrm{H}, \mathrm{ArH}), 8.53(\mathrm{~d}, J=1.5 \mathrm{~Hz}, 2 \mathrm{H}, \mathrm{ArH}), 3.94(\mathrm{~s}, 6 \mathrm{H}, \mathrm{OMe}) \mathrm{ppm} .{ }^{13} \mathrm{C}$ NMR $\left(101 \mathrm{MHz}, \mathrm{CDCl}_{3}\right): \delta=166.7$ $(\mathrm{C}=\mathrm{O}), 142.4(\mathrm{ArCH}), 132.1(\mathrm{ArCH}), 129.8\left(\mathrm{C}_{i p s o}\right), 93.4\left(\mathrm{C}_{\text {ipso }}\right), 52.6\left(\mathrm{CH}_{3}\right) \mathrm{ppm}$. 
General procedure for the synthesis of dimethyl 5-((trimethylsilyl)ethynyl)isophthalate (5):

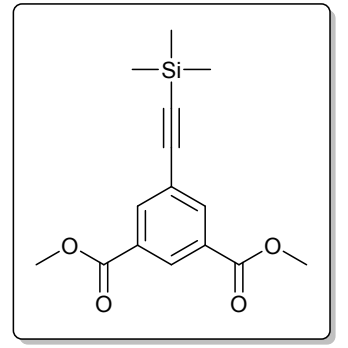

Under nitrogen atmosphere, $2.7 \mathrm{~mL}$ of ethynyltrimethylsilane $(19 \mathrm{mmol})$ were added, little by little, to a solution of dimethyl 5-iodoisophthalate 4 (5.2 g, 17mmol), CuI ( 0.6 $\mathrm{g}, 3.1 \mathrm{mmol}), \mathrm{PPh}_{3}(0.83 \mathrm{~g}, 3.2 \mathrm{mmol})$ and $\mathrm{Pd}\left(\mathrm{PPh}_{3}\right)_{2} \mathrm{Cl}_{2}(0.55 \mathrm{~g}, 0.8 \mathrm{mmol})$ in anhydrous triethylamine $(100 \mathrm{~mL})$. The reaction was left under stirring for $12 \mathrm{~h}$ at 90 ${ }^{\circ} \mathrm{C}$. After this time, the solvent was evaporated under reduced pressure and the crude obtained was purified by column chromatography ( $9: 1$ hexane/AcOEt). Compound $\mathbf{5}$ was obtained as a pale-yellow solid (yield: $80 \%, 3.9 \mathrm{~g}) .{ }^{1} \mathrm{H} \mathrm{RMN}\left(400 \mathrm{MHz}, \mathrm{CDCl}_{3}\right)$ : $\delta=8.60(\mathrm{t}, J=1.6 \mathrm{~Hz}, 1 \mathrm{H}, \mathrm{ArH}), 8.29$ (d, $J=1.6 \mathrm{~Hz}, 2 \mathrm{H}, \mathrm{ArH}), 3.94$ (s, 6H, OMe), 0.26 (s, 9H, TMS) ppm. ${ }^{13} \mathrm{C}$ RMN (101 MHz, $\left.\mathrm{CDCl}_{3}\right) \delta 165.7(\mathrm{C}=\mathrm{O}), 137.1(\mathrm{ArCH}), 131.0(\mathrm{ArCH}), 130.5\left(\mathrm{C}_{\text {ipso }}\right), 124.4\left(\mathrm{C}_{i p s o}\right), 102.9$ $\left(\mathrm{C}_{i p s o}\right), 96.9\left(\mathrm{C}_{\text {ipso }}\right), 52.7\left(\mathrm{CH}_{3}\right), 0.0$ (TMS) ppm.

\section{General procedure for the synthesis of dimethyl 5-ethynylisophthalate (6):}

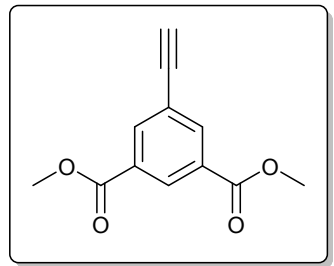

$2 \mathrm{~g}$ of $\mathrm{KF}(40.1 \mathrm{mmol})$ were added a solution of the silicon derivative 5 ( $3.9 \mathrm{~g}, 13.6$ $\mathrm{mmol})$ in $\mathrm{EtOH}(50 \mathrm{~mL})$. The reaction mixture was kept under stirring at room temperature for $4 \mathrm{~h}$. Next, the solvent was removed in vacuum. Water was added to dissolve the inorganic salts, and product was extracted three times with ethyl acetate. The organic phase was dried over anhydrous $\mathrm{Na}_{2} \mathrm{SO}_{4}$ and the solvent was removed in vacuum, obtaining precursor 6 as a light brown solid (yield: $86 \%, 2.7 \mathrm{~g}$ ). ${ }^{1} \mathrm{H}$ RMN (400 MHz, $\left.\mathrm{CDCl}_{3}\right): \delta=8.64(\mathrm{t}, J=1.6 \mathrm{~Hz}, 1 \mathrm{H}, \mathrm{ArH}), 8.32(\mathrm{~d}, J=1.6 \mathrm{~Hz}, 2 \mathrm{H}, \mathrm{ArH}), 3.95(\mathrm{~s}, 6 \mathrm{H}, \mathrm{OMe}), 3.17$ $(\mathrm{s}, 1 \mathrm{H}, \equiv \mathrm{CH}) \mathrm{ppm} .{ }^{13} \mathrm{C} \mathrm{RMN}\left(101 \mathrm{MHz}, \mathrm{CDCl}_{3}\right): \delta=165.6(\mathrm{C}=\mathrm{O}), 137.3(\mathrm{ArCH}), 131.5\left(\mathrm{C}_{\text {ipso }}\right), 130.9(\mathrm{ArCH})$, $123.4\left(\mathrm{C}_{i p s o}\right), 81.7(\equiv \mathrm{CH}), 79.4\left(\mathrm{C}_{i p s o}\right), 52.8\left(\mathrm{CH}_{3}\right) \mathrm{ppm}$.

General procedure for the synthesis of dimethyl 5-((4-(methoxycarbonyl)phenyl)ethynyl)isophthalate

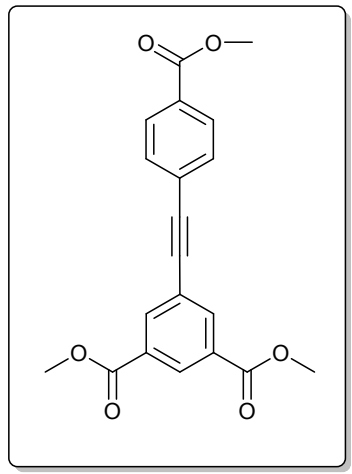

(8):

After deoxygenating $200 \mathrm{~mL}$ of triethylamine by bubbling a stream of $\mathrm{N}_{2}$, this solvent was transferred to a round-bottom flask, which contains methyl 4iodobenzoate 7 (3.53 g, $13.5 \mathrm{mmol})$, alkyne 6 ( $2.7 \mathrm{~g}, 12.3 \mathrm{mmol})$, CuI (0.115 g, 0.6 $\mathrm{mmol}), \mathrm{PPh}_{3}(0.25 \mathrm{~g}, 0.95 \mathrm{mmol})$ and $\mathrm{Pd}\left(\mathrm{PPh}_{3}\right)_{2} \mathrm{Cl}_{2}(0.24 \mathrm{~g}, 0.4 \mathrm{mmol})$. The reaction mixture was heated at $120^{\circ} \mathrm{C}$ for $12 \mathrm{~h}$ under stirring and an $\mathrm{N}_{2}$ atmosphere. After this time, the solvent was removed under reduced pressure and the reaction crude was purified by $\mathrm{SiO}_{2}$ column chromatography, (8:2 hexane/AcOEt), obtaining compound 8 as a white solid $(67 \%, 3.37 \mathrm{~g}) .{ }^{1} \mathrm{H}$ RMN $\left(400 \mathrm{MHz}, \mathrm{CDCl}_{3}\right): \delta=8.64(\mathrm{~s}, 1 \mathrm{H}, \mathrm{ArH})$, 8.37 (s, 2H, ArH), 8.04 (d, $J=7.9 \mathrm{~Hz}, 2 \mathrm{H}, \mathrm{ArH}), 7.61(\mathrm{~d}, J=7.9 \mathrm{~Hz}, 2 \mathrm{H}, \mathrm{ArH}), 3.97$ (s, 6H, OMe), 3.93 (s, 3H, OMe) ppm. $\left.{ }^{13} \mathrm{C} \mathrm{RMN} \mathrm{(101} \mathrm{MHz,} \mathrm{CDCl}_{3}\right): \delta=166.6(\mathrm{C}=\mathrm{O}), 165.7(\mathrm{C}=\mathrm{O}), 136.8$ $(\mathrm{ArCH}), 131.9(\mathrm{ArCH}), 131.3\left(\mathrm{C}_{\text {ipso }}\right), 130.7(\mathrm{ArCH}), 130.2(\mathrm{ArCH}), 129.8\left(\mathrm{C}_{i p s o}\right), 127.3\left(\mathrm{C}_{i p s o}\right), 124.0\left(\mathrm{C}_{i p s o}\right)$, $90.6\left(\mathrm{C}_{i p s o}\right), 90.3\left(\mathrm{C}_{i p s o}\right), 52.8\left(\mathrm{CH}_{3}\right), 52.5\left(\mathrm{CH}_{3}\right) \mathrm{ppm}$.

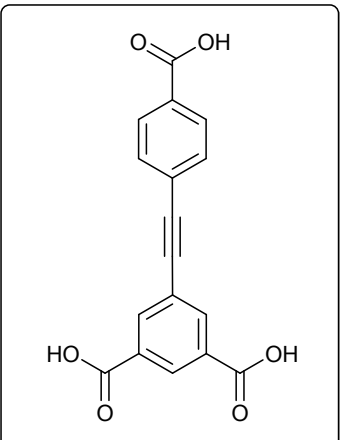

General procedure for the synthesis of 5-((4-carboxyphenyl)ethynyl) isophthalic acid $\left(\mathrm{H}_{3} \mathrm{~L}\right)$ : 
A solution of $\mathrm{NaOH}(3.286 \mathrm{~g}, 82 \mathrm{mmol})$ in $40 \mathrm{~mL}$ of $\mathrm{H}_{2} \mathrm{O}$ was added to a solution of compound $\mathbf{8}(2.88 \mathrm{~g}, 8.2$ $\mathrm{mmol}$ ) in $150 \mathrm{~mL}$ of THF. The resulting biphasic solution was stirred vigorously at $60^{\circ} \mathrm{C}$ for $24 \mathrm{~h}$, before being cooled subsequently to RT. The THF was removed under vacuum to provide typically an insoluble white solid in $\mathrm{H}_{2} \mathrm{O}$. While stirring, the aqueous layer was acidified with concentrated $\mathrm{HCl}$, precipitating a white solid. The resulting precipitate was collected by vacuum filtration and washed with ample water to provide the target compound as typically a white powder $(90 \%, 2.29 \mathrm{~g}) .{ }^{1} \mathrm{H}$ RMN $\left(400 \mathrm{MHz}, \mathrm{DMSO}-d_{6}\right) \delta=8.46(\mathrm{~s}, 1 \mathrm{H}, \mathrm{ArH})$, $8.28(\mathrm{~s}, 2 \mathrm{H}, \operatorname{ArH}), 7.99(\mathrm{~d}, J=8.2 \mathrm{~Hz}, 2 \mathrm{H}, \operatorname{ArH}), 7.75(\mathrm{~d}, J=8.1 \mathrm{~Hz}, 2 \mathrm{H}, \operatorname{ArH}) \mathrm{ppm} .{ }^{13} \mathrm{C} \mathrm{RMN}(101 \mathrm{MHz}$, DMSO- $\left.d_{6}\right) \delta=172.1(\mathrm{C}=\mathrm{O}), 171.2(\mathrm{C}=\mathrm{O}), 141.1(\mathrm{ArCH}), 137.5\left(\mathrm{C}_{i p s o}\right), 137.2(\mathrm{ArCH}), 135.0(\mathrm{ArCH}), 131.4$ $\left(\mathrm{C}_{i p s o}\right), 128.3\left(\mathrm{C}_{i p s o}\right), 95.5\left(\mathrm{C}_{i p s o}\right), 95.3\left(\mathrm{C}_{\text {ipso }}\right) \mathrm{ppm}$.

Single crystal preparation of GR-MOF-6: $10 \mathrm{mg}(0.033 \mathrm{mmol})$ of $\mathrm{YCl}_{3} 6 \mathrm{H}_{2} \mathrm{O}$ was dissolved in $0.5 \mathrm{~mL}$ of $\mathrm{N}, \mathrm{N}$-dimethylformamide (DMF). Then, $0.5 \mathrm{~mL}$ of water was added to the yttrium solution. In a separated vial, $10 \mathrm{mg}(0.033 \mathrm{mmol})$ of linker $\mathrm{H}_{3} \mathrm{~L}$ was solved by heating in $1 \mathrm{~mL}$ of DMF. The metal solution was added dropwise to the ligand solution, and the resulting colourless mixture was placed in a closed glass vessel and heated in an oven at $95^{\circ} \mathrm{C}$ for $24 \mathrm{~h}$. X-ray quality crystals of GR-MOF-6 were obtained during heating process under autogenous pressure. Elemental Analysis calculated. for $\mathrm{C}_{49} \mathrm{H}_{49} \mathrm{~N}_{5} \mathrm{O}_{17} \mathrm{Y}_{2}: \mathrm{C}, 50.83 ; \mathrm{H}, 4.27 ; \mathrm{N}, 6.05$. Found: $\mathrm{C}, 50.79 ; \mathrm{H}, 4.19 ; \mathrm{N}, 6.11$. In addition to the elemental analyses, the purity of all the samples was checked by FT-IR spectra. It should be noted that the same material was obtained when changing the yttrium chloride by yttrium nitrate or yttrium(III) trifluoromethanesulfonate. Yield based on metal: $64 \pm 6 \%$.

General procedure for the cyanosilylation reaction: $\mathrm{In}$ a $1 \mathrm{~mL}$ vial with a septum screw capped equipped with a stirring bar, it was weighed GR-MOF-6 catalyst (1.35 mg, $0.5 \mathrm{~mol} \%$ ). Subsequently, the corresponding amount of carbonylic compound $1(0.5 \mathrm{mmol})$ was added followed by TMSCN $(69 \mu \mathrm{L}, 0.55 \mathrm{mmol}, 1.1$ equiv.) and the reaction was stirred under inert $\mathrm{N}_{2}$ atmosphere at room temperature during the corresponding time. Once the reaction was finished the catalyst was removed by centrifugation ( $8000 \mathrm{rpm}, 3 \mathrm{~min}$ ) and washed with DCM $(2 \times 0.5 \mathrm{~mL})$ obtaining the corresponding pure product 3 after removal of the solvent with rotary evaporator. When not fully conversion was reached the product was purified by column chromatography using hexane as eluent.

General procedure for the gram scale reaction: In a $3 \mathrm{~mL}$ vial equipped with septum and stirring bar, it was weighed GR-MOF-6 catalyst (13.5 mg, $0.5 \mathrm{~mol} \%)$. Then, it was added benzaldehyde 1a (507 $\mu \mathrm{L}, 5 \mathrm{mmol}$ ) followed by TMSCN ( $688 \mu \mathrm{L}, 5.5 \mathrm{mmol}, 1.1$ equiv.) and the reaction was stirred under inert $\mathrm{N}_{2}$ atmosphere at room temperature for $7.5 \mathrm{~h}$. Once the reaction was finished the catalyst was removed by centrifugation ( 8000 $\mathrm{rpm}, 3 \mathrm{~min})$ and washed with DCM $(2 \times 2 \mathrm{~mL})$ obtaining the corresponding pure product $\mathbf{2 a}$ after removal of the solvent with rotary evaporator with $95 \%$ isolated yield $(977.6 \mathrm{mg})$.

Quantitative NMR acquisition parameters. ${ }^{1} \mathrm{H}$ NMR determination of product conversion was carried out by comparing signals arising from both $\mathrm{CH}$ of aldehyde $\mathbf{1}$ and cyanosilylated product $\mathbf{2}$. The standard acquisition parameters were one-dimensional pulse sequence which includes a $30^{\circ}$ flip angle (Bruker zg30), recycle time $(\mathrm{D} 1=30 \mathrm{~s})$, time domain $(\mathrm{TD}=27 \mathrm{k})$, number of scans $(\mathrm{NS}=32)$, acquisition time $(\mathrm{AQ}=2.05$ $\mathrm{s})$, transmitter (frequency) offset $(\mathrm{O} 1 \mathrm{P}=6.0 \mathrm{ppm})$, and spectral width $(\mathrm{SW}=22.0 \mathrm{ppm})$.

Recyclability of the catalyst: In a $1 \mathrm{ml}$ vial with a septum screw capped equipped with a stirring bar, it was weighed the catalysts $(4.05 \mathrm{mg}, 0.5 \mathrm{~mol} \%)$. Then, the corresponding amount of benzaldehyde $1 \mathrm{a}(152 \mu \mathrm{L}, 1.5$ mmol) followed by TMSCN (206 $\mu \mathrm{L}, 1.65 \mathrm{mmol}, 1.1$ equiv.) was added and the reaction was stirred under inert $\mathrm{N}_{2}$ atmosphere at room temperature during $7.5 \mathrm{~h}$. After this time, $1.5 \mathrm{~mL}$ of DCM was added to the 
reaction mixture and transferred to a centrifuge tube for the separation of the catalyst. The centrifugation of the mixture was carried out at $8000 \mathrm{rpm}$ during $3 \mathrm{~min}$. After that, the solution was eliminated, and the GRMOF- 6 catalyst was washed with DCM $(2 \times 1.5 \mathrm{~mL})$. Later, the catalyst was dried under vacuum and reused in the next cycle of the reaction with the same reaction conditions previously described. 


\section{Crystallographic data}

Table S1. Crystallographic data and structure refinement details of compound GR-MOF-6.

\begin{tabular}{|c|c|}
\hline Compound & GR-MOF-6 \\
\hline Formula & $\mathrm{C}_{49} \mathrm{H}_{49} \mathrm{~N}_{5} \mathrm{O}_{17} \mathrm{Y}_{2}$ \\
\hline$M_{r}$ & 1157.75 \\
\hline Crystal system & Monoclinic \\
\hline Space group (no.) & $C 2 / \mathrm{c}$ \\
\hline$a(\AA)$ & $29.4718(12)$ \\
\hline$b(\AA)$ & $14.1443(6)$ \\
\hline$c(\AA)$ & $13.7369(7)$ \\
\hline$\alpha\left(^{\circ}\right)$ & 90 \\
\hline$\beta\left(^{\circ}\right)$ & $99.969(2)$ \\
\hline$\gamma\left({ }^{\circ}\right)$ & 90 \\
\hline$V\left(\AA^{3}\right)$ & $5639.9(4)$ \\
\hline $\mathrm{Z}$ & 4 \\
\hline$\rho_{\text {calc }} \mathrm{g} / \mathrm{cm}^{3}$ & 1.363 \\
\hline $\mathrm{F}(000)$ & 2368 \\
\hline Radiation & $\operatorname{MoK} \alpha(\lambda=0.71073)$ \\
\hline Index ranges & $-33 \leq \mathrm{h} \leq 44,-20 \leq \mathrm{k} \leq 19,-19 \leq 1 \leq 19$ \\
\hline Independent reflections & $31400\left[\mathrm{R}_{\mathrm{int}}=0.0492\right]$ \\
\hline dness-of-fit on $\mathrm{F}^{2}$ & 1.033 \\
\hline $1 \mathrm{R}$ indexes $[\mathrm{I}>=2 \sigma(\mathrm{I})]$ & $\mathrm{R}_{1}=0.0551, \mathrm{wR}_{2}=0.1520$ \\
\hline $1 \mathrm{R}$ indexes [all data] & $\mathrm{R}_{1}=0.0877, \mathrm{wR}_{2}=0.1710$ \\
\hline sest diff. peak/hole / e $\AA^{-3}$ & $1.276 /-0.936$ \\
\hline
\end{tabular}


4. Selected bond lengths and angles data

Table S2. Table of the selected bond lengths $(\AA)$ and angles $\left(^{\circ}\right)$ for compound GR-MOF-6.

\begin{tabular}{lll}
\hline GR-MOF-6 & Distances \\
\hline Y1 & O1 & $2.333(3)$ \\
Y1 & O3 & $2.357(2)$ \\
Y1 & O8 & $2.389(2)$ \\
Y2 & O9 & $2.304(2)$ \\
Y2 & O21 & $2.469(3)$ \\
Y2 & O22 & $2.401(3)$ \\
Y2 & O23A & $2.298(13)$ \\
Y2 & O28A & $2.381(18)$ \\
\hline
\end{tabular}

\begin{tabular}{ll}
\hline GR-MOF-6 & Angles \\
\hline O1 Y1 O3 & $132.55(9)$ \\
O1 Y1 O8 & $75.23(9)$ \\
O1 Y1 O9 & $68.20(8)$ \\
O1 Y1 C20 & $72.07(10)$ \\
O1 Y1 O21 & $71.57(10)$ \\
O1 Y1 O22 & $77.50(10)$ \\
O3 Y1 O8 & $86.97(9)$ \\
O3 Y1 O9 & $66.64(8)$ \\
O3 Y1 C20 & $155.17(11)$ \\
O3 Y1 O21 & $143.99(10)$ \\
O3 Y1 O22 & $143.76(10)$ \\
O8 Y1 O9 & $50.41(7)$ \\
O8 Y1 C20 & $105.59(10)$ \\
O8 Y1 O21 & $128.52(9)$ \\
O8 Y1 O22 & $82.16(10)$ \\
O9 Y1 O1 & $74.37(9)$ \\
O9 Y1 O3 & $80.86(8)$ \\
O9 Y1 O8 & $124.35(9)$ \\
O9 Y1 O9 & $75.21(8)$ \\
O9 Y1 C20 & $107.72(10)$ \\
O9 Y1 C20 & $137.64(10)$ \\
O9 Y1 O21 & $82.46(9)$ \\
O9 Y1 O22 & $133.13(9)$ \\
O21 Y1 O9 & $137.84(10)$ \\
O21 Y1 C20 & $26.48(10)$ \\
O22 Y1 O9 & $126.30(10)$ \\
O22 Y1 C20 & $26.69(11)$ \\
O22 Y1 O21 & $53.15(9)$ \\
\hline
\end{tabular}




\section{Experimental PXRD}

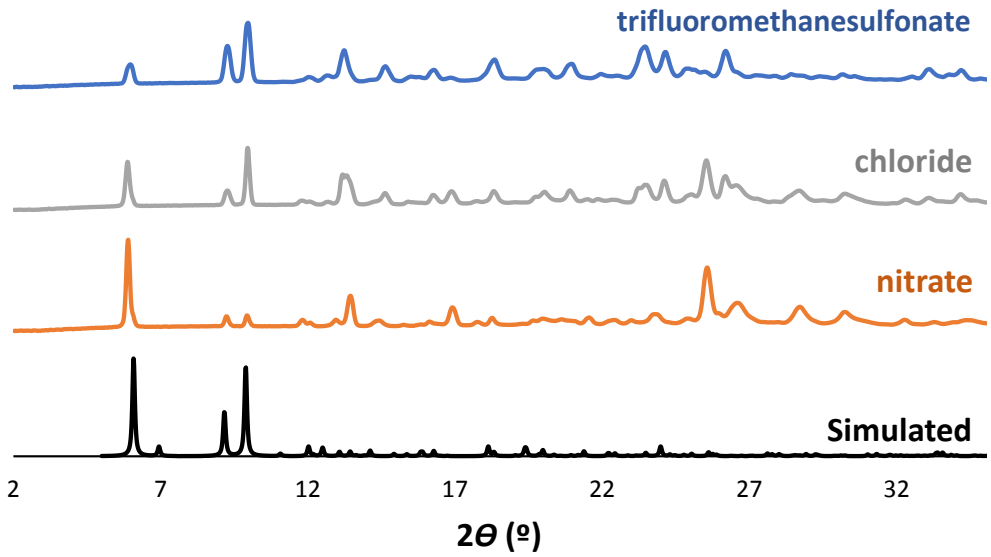

Figure S1. Effect of the Y(III) precursor used in the synthesis of GR-MOF-6 over the crystallinity of the framework. 
6. FTIR analysis of $\mathrm{H}_{3} \mathrm{~L}$ ligand and GR-MOF-6

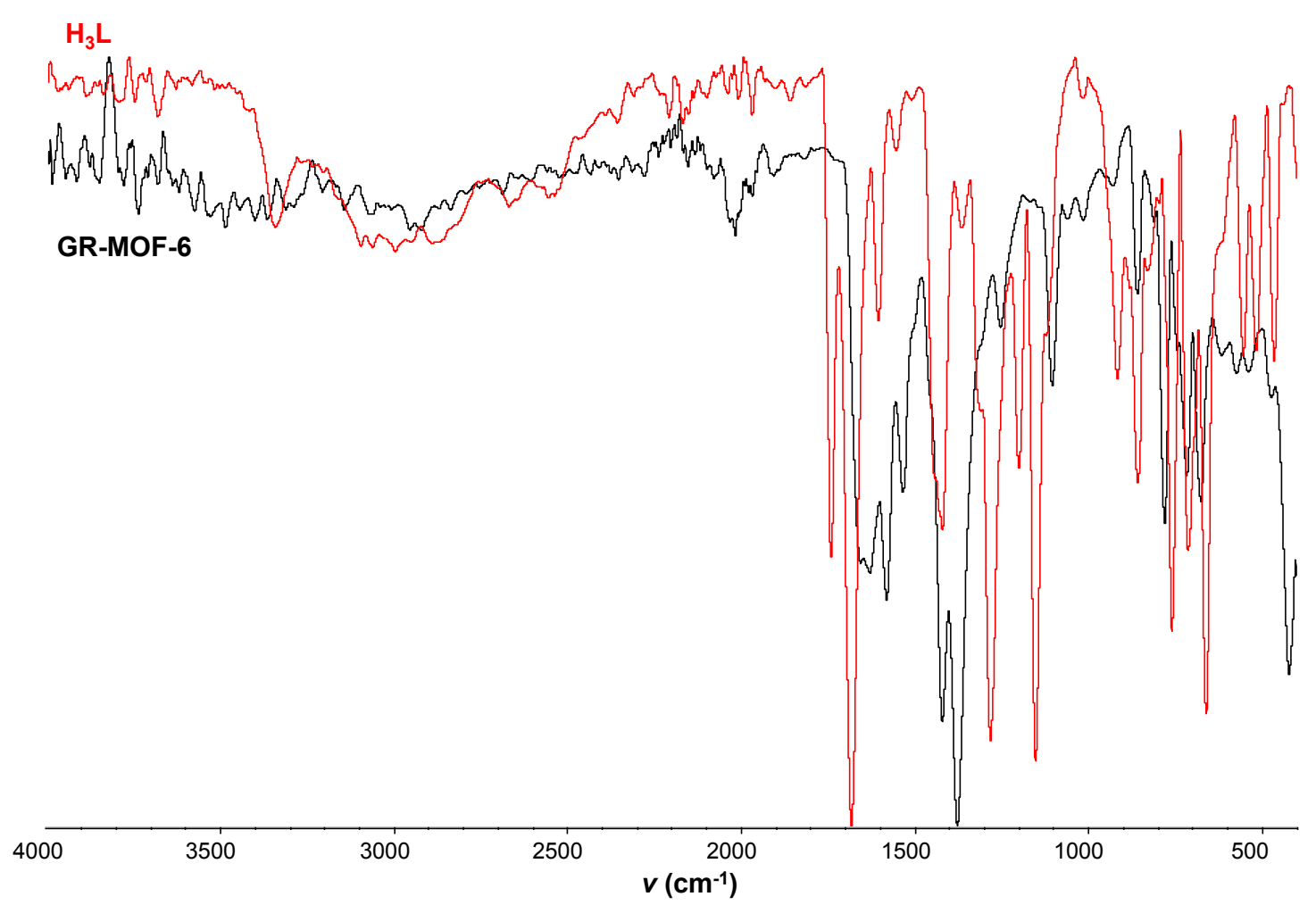

Figure S2. FTIR (ATR mode) spectra of GR-MOF-6 (black) and $\mathrm{H}_{3} \mathrm{~L}$ (red).

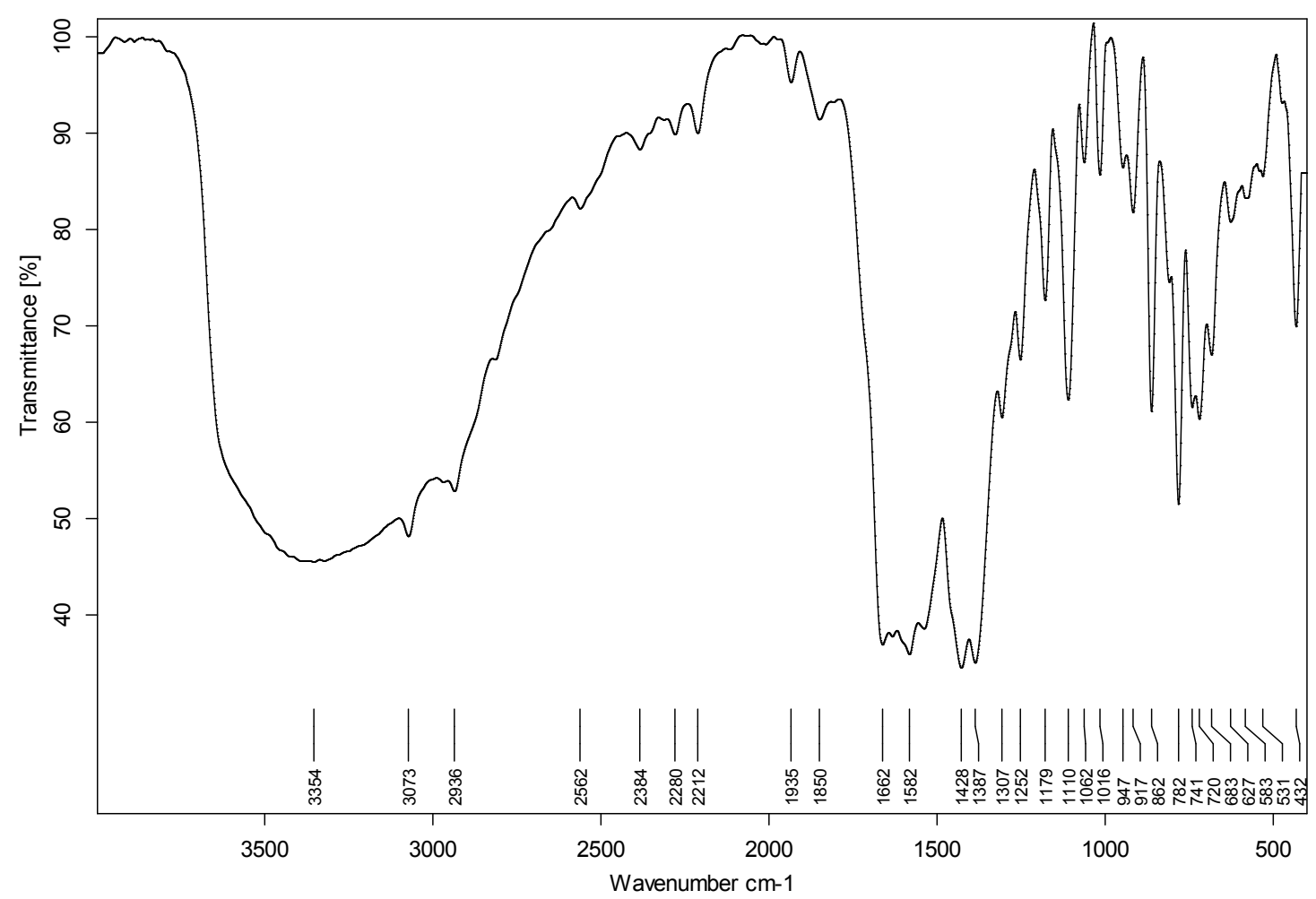

Figure S3. FTIR (DRIFT mode) spectrum of GR-MOF-6. 


\section{Thermal analysis}

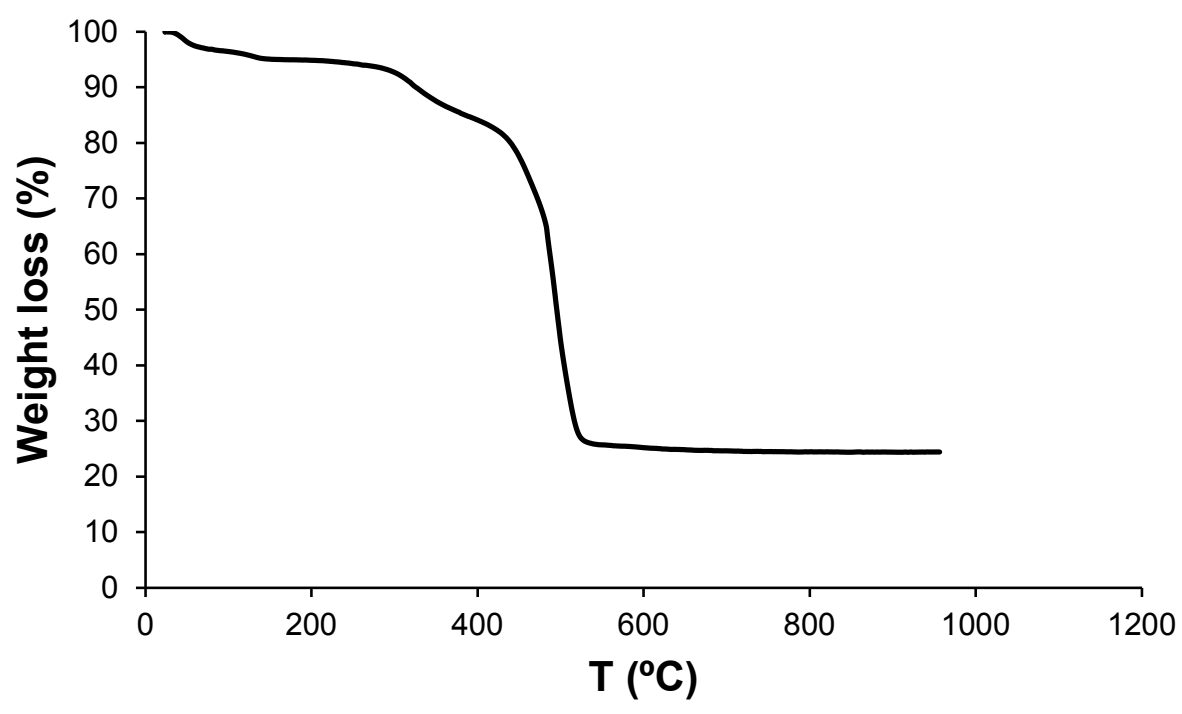

Figure S4. TGA for pristine GR-MOF-6.

\section{Adsorption properties}

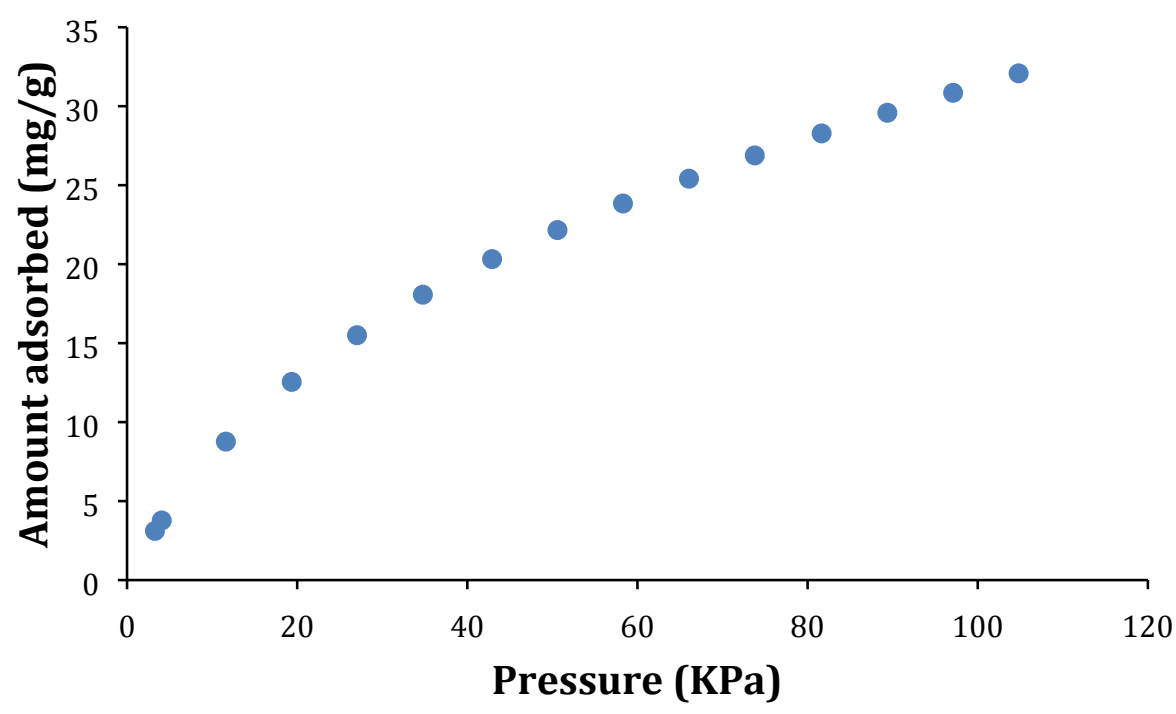

Figure S5. $\mathrm{CO}_{2}$ sorption isotherm at $273 \mathrm{~K}$. 


\section{Additional views of the structure}

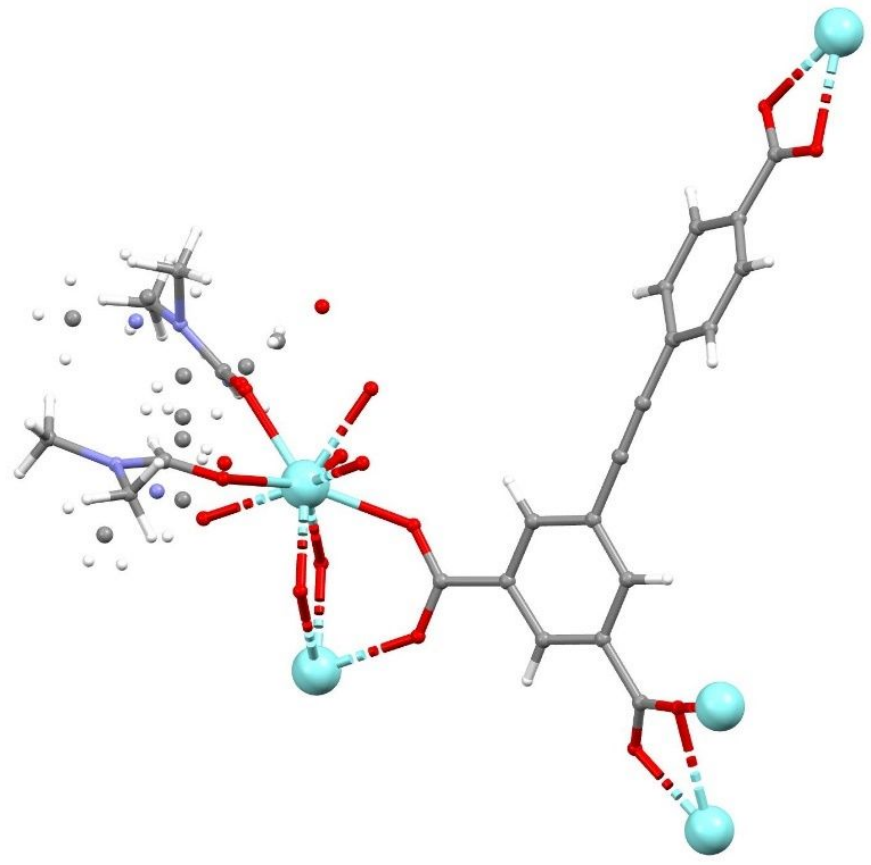

Figure S6. Perspective of GR-MOF-6 in which two coordinated DMF molecules disordered into two positions are shown.

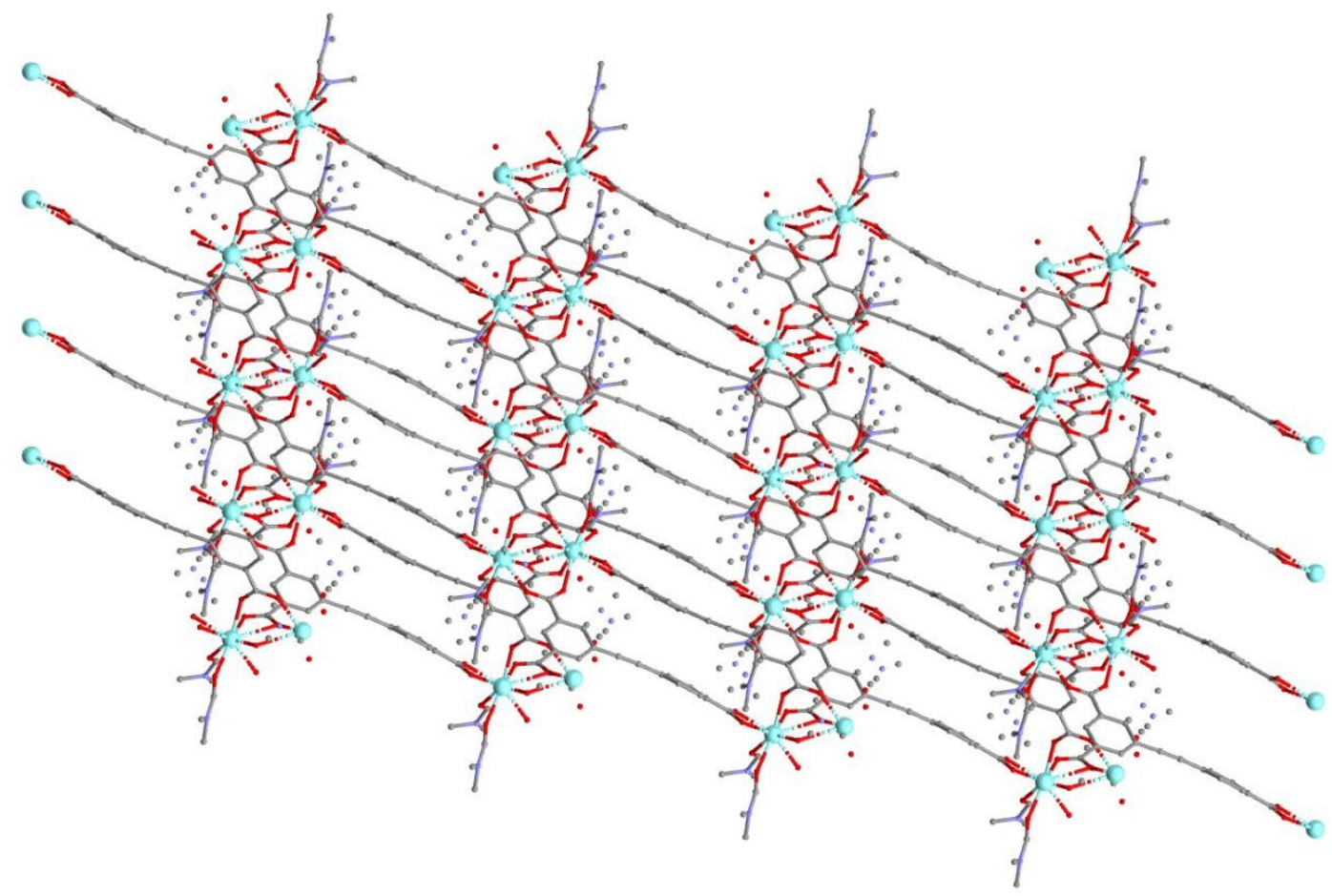

Figure S7. View along b axis in which can be observed that the polytopic ligand, due to his planarity, imposes an interesting possible corrugated arrangement. 


\section{Study of $\zeta$-potential at different $\mathrm{pH}$ values and size distribution}

Table S3. Variation of $\zeta$-potential at different $\mathrm{pH}$ values.

\begin{tabular}{|c|c|c|}
\hline $\boldsymbol{p H}$ & Electrophoretic mobility $(\boldsymbol{\mu m} \cdot \mathbf{c m} / \boldsymbol{V} \cdot \mathbf{s})$ & Zeta-potential $(\mathbf{m} \boldsymbol{V})$ \\
\hline 4 & $0.429 \pm 0.01$ & $2.85 \pm 0.4$ \\
\hline 5 & $-0.597 \pm 0.02$ & $-7.61 \pm 0.2$ \\
\hline 6 & $-0.630 \pm 0.03$ & $-8.04 \pm 0.4$ \\
\hline 7 & $-1.032 \pm 0.02$ & $-13.2 \pm 0.3$ \\
\hline 8 & $-1.118 \pm 0.02$ & $-14.3 \pm 0.3$ \\
\hline 9 & $-1.520 \pm 0.03$ & $-19.4 \pm 0.3$ \\
\hline 10 & $-1.553 \pm 0.02$ & $-19.8 \pm 0.3$ \\
\hline
\end{tabular}
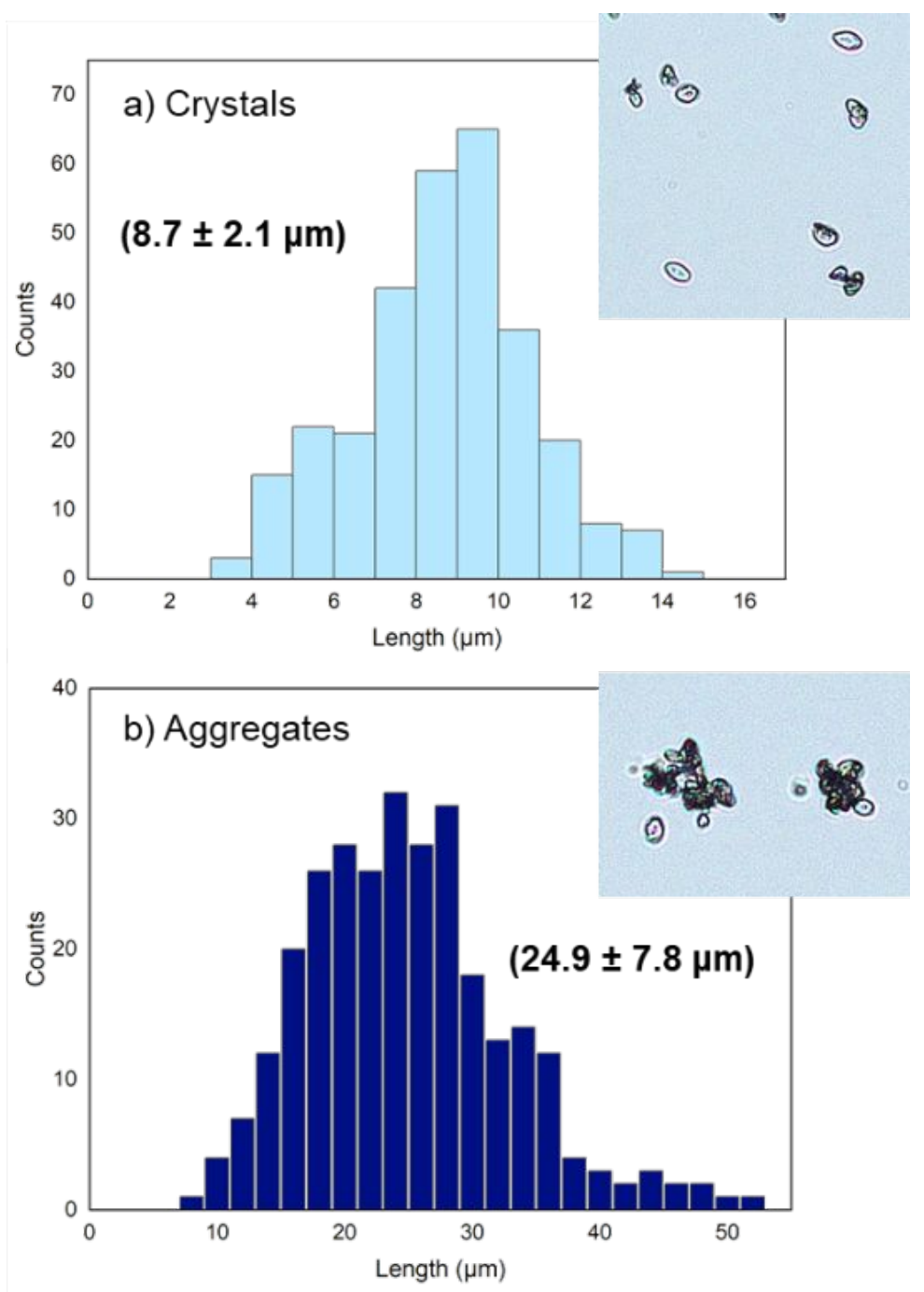

Figure S8. Size distribution of the MOF crystals in the fraction steadily suspended in water (about a $40 \%$ of the total amount), determined from optical microscope images. (a) Average size of individual crystals and (b) average size of aggregates. 


\section{Optimization of the reaction conditions}

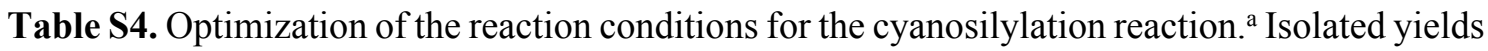
are given in brackets.

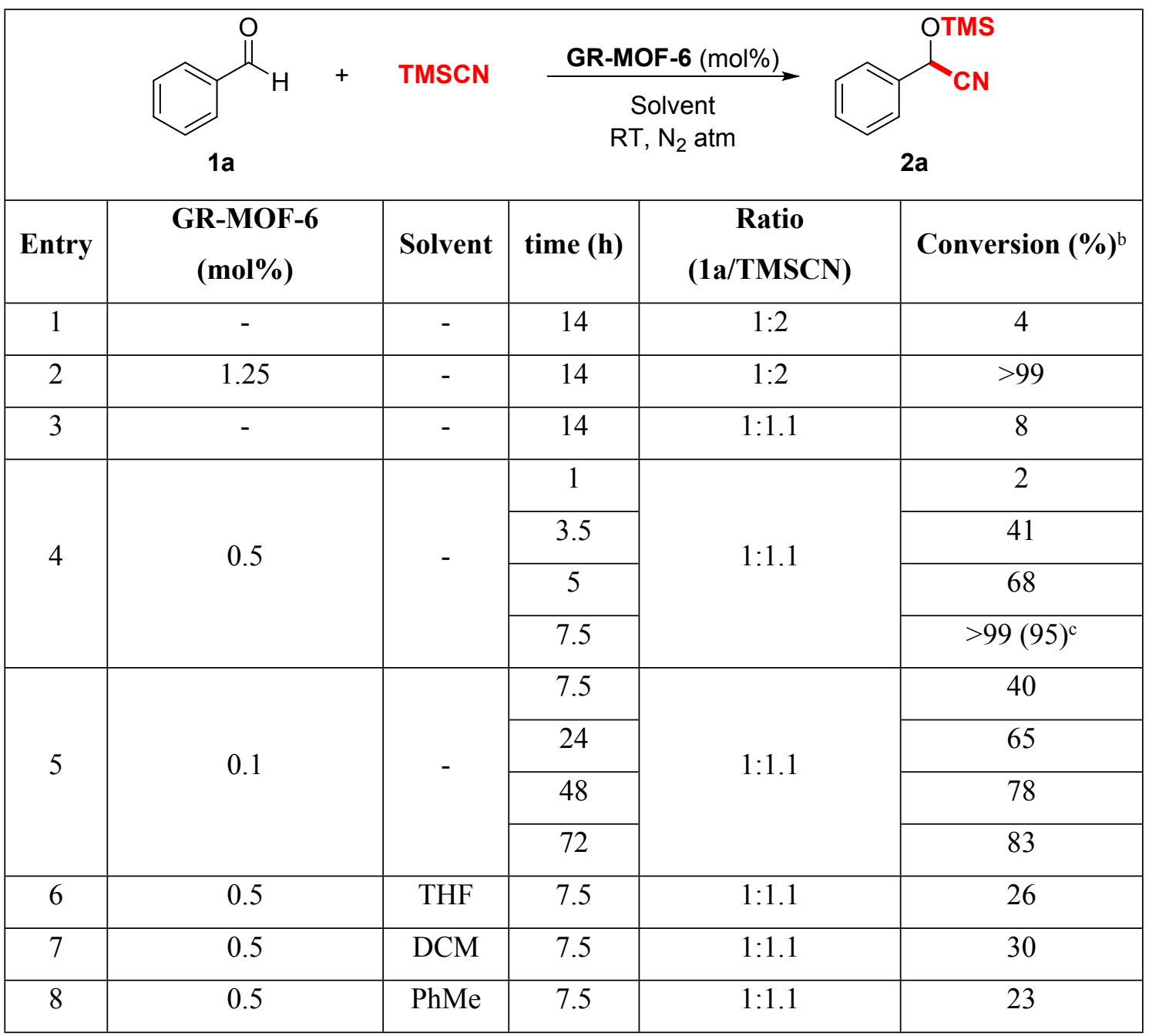

a Reaction carried out using substrates 1a (51 $\mu \mathrm{L}, 0.5 \mathrm{mmol})$ and TMSCN $(69 \mu \mathrm{L}, 0.55 \mathrm{mmol}$, 1.1 equiv.) under inert $\mathrm{N}_{2}$ atmosphere at room temperature. ${ }^{\mathrm{b}}$ Conversions (relative to aldehyde 1a) determined by ${ }^{1} \mathrm{H}$ NMR of the reaction crude. ${ }^{\mathrm{c}}$ Multigram scale reaction carried out with 507 $\mu \mathrm{L}$ ( $5 \mathrm{mmol}$ ) of substrate $1 \mathrm{a}$ and $688 \mu \mathrm{L}$ (5.5 mmol, 1.1 equiv.) of TMSCN. 
12. TOF using $0.1 \mathrm{~mol} \%$ of GR-MOF-6

$0.1 \mathrm{~mol} \%$ GR-MOF-6

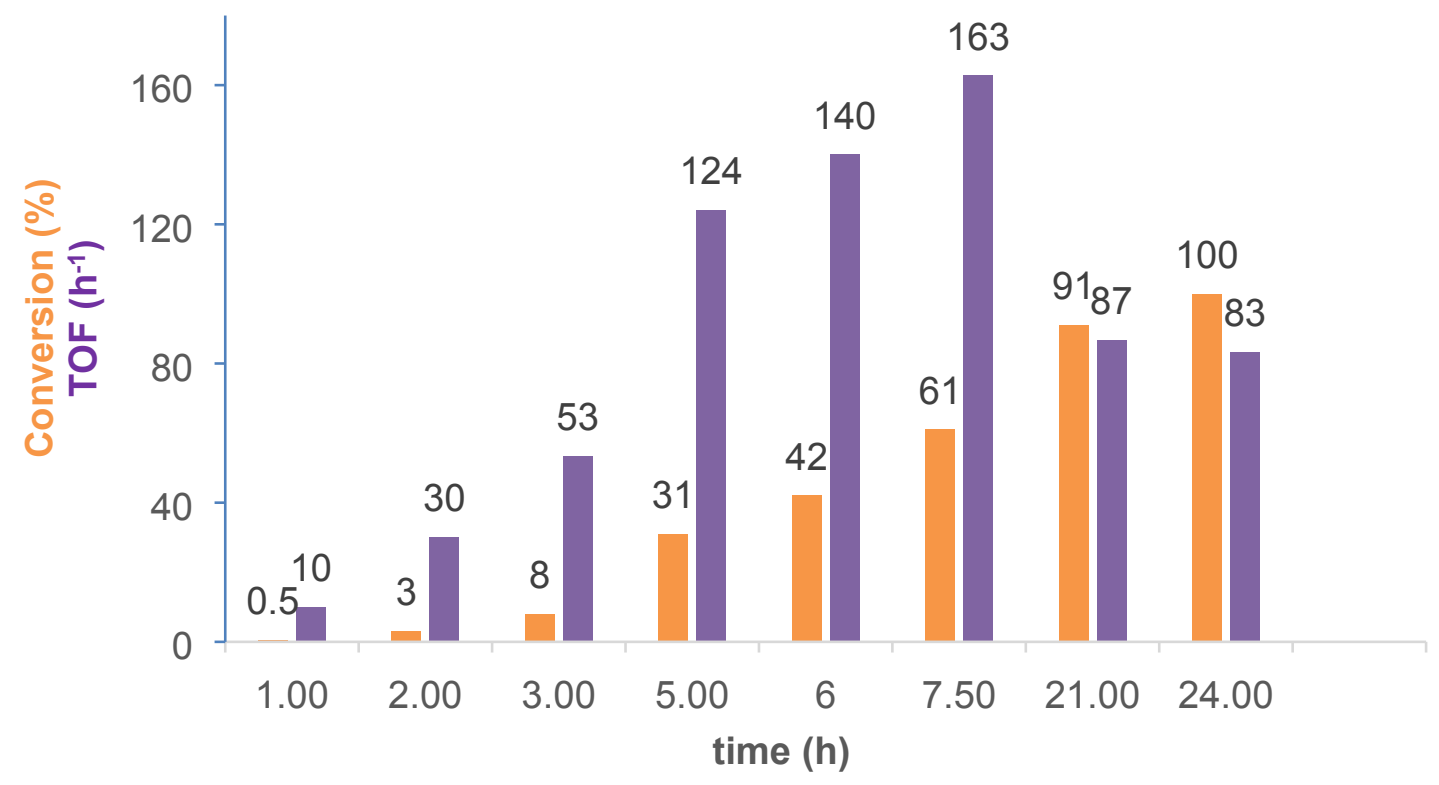

Figure S9. Conversion and TOF of the reaction at different times using $0.1 \mathrm{~mol} \%$ of GR-MOF6. 


\section{Characterization Data of Products}<smiles>N#CC(O[Na])c1ccccc1</smiles>

2-Phenyl-2-((trimethylsilyl)oxy)acetonitrile (2a). This product has been previously reported. ${ }^{7}$

${ }^{1}$ H NMR (300.13 MHz, CDCl 3 ): $\delta$ 7.50-7.35 (m, 5H, ArH), 5.50 (s, 1H, CHCN), 0.23 (s, 9H, TMS) ppm.

${ }^{13}$ C NMR (75.48 MHz, CDCl 3 ): $\delta 136.2\left(\mathrm{C}_{i p s o}\right), 129.3(\mathrm{ArCH}), 128.9(\mathrm{ArCH}), 126.3(\mathrm{ArCH}), 119.1(\mathrm{CN})$, $63.6(\mathrm{CH}),-0.29$ (TMS) ppm.

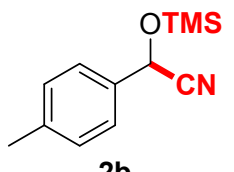

$2 b$

2-(p-Tolyl)-2-((trimethylsilyl)oxy)acetonitrile (2b). This product has been previously reported. ${ }^{8}$

${ }^{1} \mathbf{H}$ NMR (300.13 MHz, CDCl 3 ): $\delta 7.37(\mathrm{~d}, J=7.9 \mathrm{~Hz}, 2 \mathrm{H}, \mathrm{ArH}), 7.22(\mathrm{~d}, J=7.9 \mathrm{~Hz}, 2 \mathrm{H}, \mathrm{ArH}), 5.47$ (s, $1 \mathrm{H}, \mathrm{CHCN}), 2.38$ (s, 3H, CH3), 0.23 (s, 9H, TMS) ppm.

${ }^{13}$ C NMR (75.48 MHz, $\left.\mathbf{C D C l}_{3}\right): \delta 139.3\left(\mathrm{C}_{i p s o}\right), 133.3\left(\mathrm{C}_{i p s o}\right), 129.5(\mathrm{ArCH}), 126.3(\mathrm{ArCH}), 119.2(\mathrm{CN})$, $63.5(\mathrm{CH}), 21.1(\mathrm{CH} 3),-0.29(\mathrm{TMS}) \mathrm{ppm}$.<smiles>COc1ccc(C(C#N)OC)cc1</smiles>

2-(4-Methoxyphenyl)-2-((trimethylsilyl)oxy)acetonitrile (2c). This product has been previously reported. ${ }^{9}$

${ }^{1} \mathrm{H}$ NMR (300.13 MHz, $\left.\mathbf{C D C l}_{3}\right): \delta 7.38(\mathrm{~d}, J=8.6 \mathrm{~Hz}, 2 \mathrm{H}, \mathrm{ArH}), 6.92(\mathrm{~d}, J=8.6 \mathrm{~Hz}, 2 \mathrm{H}, \mathrm{ArH}), 5.43(\mathrm{~s}$, $1 \mathrm{H}, \mathrm{CHCN}), 3.82$ (s, 3H, OMe), -0.21 (s, 9H, TMS) ppm.

${ }^{13}$ C NMR (75.48 MHz, CDCl $\left._{3}\right): \delta 160.3\left(\mathrm{C}_{i p s o}\right), 128.3\left(\mathrm{C}_{i p s o}\right), 127.9(\mathrm{ArCH}), 119.3(\mathrm{CN}), 114.2(\mathrm{ArCH})$, $63.3(\mathrm{CH}), 55.3\left(\mathrm{OCH}_{3}\right),-0.24(\mathrm{TMS}) \mathrm{ppm}$. 
2d

2-(4-(Dimethylamino)phenyl)-2-((trimethylsilyl)oxy)acetonitrile (2d).

${ }^{1} \mathbf{H}$ NMR (300.13 MHz, $\left.\mathbf{C D C l}_{3}\right): \delta 7.31(\mathrm{~d}, J=8.7 \mathrm{~Hz}, 2 \mathrm{H}, \mathrm{ArH}), 6.71(\mathrm{~d}, J=8.7 \mathrm{~Hz}, 2 \mathrm{H}, \mathrm{ArH}), 5.39$ (s, $1 \mathrm{H}, \mathrm{CHCN}), 2.98$ (s, 6H, $\mathrm{NMe}_{2}$ ), 0.19 (s, 9H, TMS) ppm.

${ }^{13}$ C NMR (75.48 MHz, $\left.\mathbf{C D C l}_{3}\right): \delta 151.1\left(\mathrm{C}_{i p s o}\right), 127.8(\mathrm{ArCH}), 123.6\left(\mathrm{C}_{i p s o}\right), 119.6(\mathrm{CN}), 112.1(\mathrm{ArCH})$, $63.7(\mathrm{CH}), 40.3\left(\mathrm{CH}_{3}\right),-0.16(\mathrm{TMS}) \mathrm{ppm}$.

IR (ATR): $v 2959\left(\mathrm{CH}_{3}\right), 2230(\mathrm{C} \equiv \mathrm{N}), 1621(\mathrm{C}=\mathrm{N}), 1531(\mathrm{C}=\mathrm{C}), 1371(\mathrm{C}-\mathrm{N}), 1257(\mathrm{C}-\mathrm{O}), 1061(\mathrm{C}-\mathrm{O})$, $835 \mathrm{~cm}^{-1}$.

Elemental Analysis calc. for $\mathrm{C}_{13} \mathrm{H}_{20} \mathrm{~N}_{2} \mathrm{OSi}$ : C 62.86, N, 11.28, H 8.12; found: C 63.23, N 11.65, H 7.67.

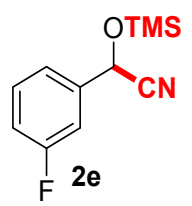

2-(3-Fluorophenyl)-2-((trimethylsilyl)oxy)acetonitrile (2e). This product has been previously reported..$^{10}$

${ }^{1} \mathbf{H}$ NMR (300.13 MHz, $\left.\mathbf{C D C l}_{3}\right): \delta 7.45-7.35(\mathrm{~m}, 1 \mathrm{H}, \mathrm{ArH}), 7.25-7.15(\mathrm{~m}, 2 \mathrm{H}, \mathrm{ArH}), 7.10-7.05(\mathrm{~m}, 1 \mathrm{H}$, $\mathrm{ArH}), 5.50$ (s, 1H, CHCN), 0.25 (s, 9H, TMS) ppm.

${ }^{13}$ C NMR (75.48 MHz, $\left.\mathbf{C D C l}_{3}\right): \delta 162.8\left(\mathrm{~d}, \mathrm{C}_{i p s o},{ }^{1} J_{\mathrm{C}-\mathrm{F}}=247.7 \mathrm{~Hz},\right), 138.6\left(\mathrm{~d}, \mathrm{C}_{i p s o},{ }^{3} J_{\mathrm{C}-\mathrm{F}}=7.8 \mathrm{~Hz},\right), 130.5$ $\left(\mathrm{d}, \mathrm{ArCH},{ }^{3} J_{\mathrm{C}-\mathrm{F}}=8.1 \mathrm{~Hz},\right), 121.7\left(\mathrm{~d}, \mathrm{ArCH},{ }^{4} J_{\mathrm{C}-\mathrm{F}}=3.0 \mathrm{~Hz}\right), 118.7(\mathrm{CN}), 116.3\left(\mathrm{~d}, \mathrm{ArCH},{ }^{2} J_{\mathrm{C}-\mathrm{F}}=21.2 \mathrm{~Hz}\right)$, $113.3\left(\mathrm{~d}, \mathrm{ArCH},{ }^{2} J_{\mathrm{C}-\mathrm{F}}=23.4 \mathrm{~Hz}\right), 62.8\left(\mathrm{~d}, \mathrm{CH},{ }^{4} J_{\mathrm{C}-\mathrm{F}}=2.1 \mathrm{~Hz}\right),-0.4(\mathrm{TMS}) \mathrm{ppm}$.

${ }^{19}$ F-NMR (282.4 MHz, $\mathrm{CDCl}_{3}$ ): $\delta-111.4 \mathrm{ppm}$.<smiles>COC(C#N)c1ccc(Cl)cc1</smiles>

2-(4-Chlorophenyl)-2-((trimethylsilyl)oxy)acetonitrile (2f). This product has been previously reported. ${ }^{11}$ ${ }^{1}$ H NMR (300.13 MHz, CDCl $)$ ): $\delta$ 7.45-7.40 (m, 5H, ArH), 5.49 (s, 1H, CHCN), 0.26 (s, 9H, TMS) ppm.

${ }^{13}$ C NMR (75.48 MHz, $\left.\mathbf{C D C l}_{3}\right): \delta 135.3\left(\mathrm{C}_{i p s o}\right), 134.8\left(\mathrm{C}_{i p s o}\right), 129.1(\mathrm{ArCH}), 127.7(\mathrm{ArCH}), 118.8(\mathrm{CN})$, $63.0(\mathrm{CH}),-0.30$ (TMS) ppm. 
(2g

2-(4-Nitrophenyl)-2-((trimethylsilyl)oxy)acetonitrile (2g). This product has been previously reported. ${ }^{11}$

${ }^{1}$ H NMR (300.13 MHz, CDCl $)$ ): $\delta 8.29(\mathrm{~d}, J=8.8 \mathrm{~Hz}, 2 \mathrm{H}, \mathrm{ArH}), 7.67(\mathrm{~d}, J=8.8 \mathrm{~Hz}, 2 \mathrm{H}, \mathrm{ArH}), 5.59$ (s, $1 \mathrm{H}, \mathrm{CHCN}), 0.29$ (s, 9H, TMS) ppm.

${ }^{13}$ C NMR (75.48 MHz, CDCl $\left._{3}\right): \delta 148.4\left(\mathrm{C}_{i p s o}\right), 142.8\left(\mathrm{C}_{i p s o}\right), 127.0(\mathrm{ArCH}), 124.1(\mathrm{ArCH}), 118.1(\mathrm{CN})$, $62.6(\mathrm{CH}),-0.40(\mathrm{TMS}) \mathrm{ppm}$.<smiles>COC(C#N)c1ccccn1</smiles>

2-(Pyridin-2-yl)-2-((trimethylsilyl)oxy)acetonitrile (2h). This product has been previously reported. ${ }^{12}$

${ }^{1}$ H NMR (300.13 MHz, CDCl $)_{3}$ : $\delta 8.60-8.55(\mathrm{~m}, 1 \mathrm{H}, \mathrm{ArH}), 7.79(\mathrm{dt}, J=7.7,1.7 \mathrm{~Hz}, 1 \mathrm{H}, \mathrm{ArH}), 7.59(\mathrm{~d}, J$ $=7.7 \mathrm{~Hz}, 1 \mathrm{H}, \mathrm{ArH}), 7.35-7.25(\mathrm{~m}, 1 \mathrm{H}, \mathrm{ArH}), 5.58(\mathrm{~s}, 1 \mathrm{H}, \mathrm{CHCN}), 0.26(\mathrm{~s}, 9 \mathrm{H}, \mathrm{TMS}) \mathrm{ppm}$.

${ }^{13}$ C NMR (75.48 MHz, $\left.\mathbf{C D C l}_{3}\right): \delta 155.4\left(\mathrm{C}_{i p s o}\right), 149.3(\mathrm{ArCH}), 137.5(\mathrm{ArCH}), 124.0(\mathrm{ArCH}), 120.5$ (ArCH), $118.6(\mathrm{CN}), 65.1(\mathrm{CH}),-0.37$ (TMS) ppm.<smiles>COC(C#N)c1ccc2ccccc2n1</smiles>

2-(Quinolin-2-yl)-2-((trimethylsilyl)oxy)acetonitrile (2i).

${ }^{1} \mathbf{H}$ NMR (300.13 MHz, $\left.\mathbf{C D C l}_{3}\right): \delta 8.27(\mathrm{~d}, J=8.5 \mathrm{~Hz}, 1 \mathrm{H}, \mathrm{ArH}), 8.09(\mathrm{~d}, J=8.5 \mathrm{~Hz}, 1 \mathrm{H}, \mathrm{ArH}), 7.85(\mathrm{~d}$, $J=8.1 \mathrm{~Hz}, 1 \mathrm{H}, \mathrm{ArH}), 7.80-7.70$ (m, 2H, ArH), 7.60-7.55 (m, 1H, ArH), 5.75 (s, 1H, CHCN), 0.27 (s, TMS) ppm.

${ }^{13}$ C NMR (75.48 MHz, $\left.\mathbf{C D C l}_{3}\right): \delta 155.4\left(\mathrm{C}_{i p s o}\right), 147.2\left(\mathrm{C}_{i p s o}\right), 137.9(\mathrm{ArCH}), 130.1(\mathrm{ArCH}), 129.3(\mathrm{ArCH})$, $128.0\left(\mathrm{C}_{\text {ipso }}\right), 127.6(\mathrm{ArCH}), 127.3(\mathrm{ArCH}), 118.6(\mathrm{CN}), 117.8(\mathrm{ArCH}), 65.9(\mathrm{CH}),-0.31(\mathrm{TMS}) \mathrm{ppm}$.

IR (ATR): $v 3058\left(\mathrm{C}_{\mathrm{sp}}{ }^{2}-\mathrm{H}\right), 2959\left(\mathrm{CH}_{3}\right), 2170(\mathrm{C} \equiv \mathrm{N}), 1593(\mathrm{C}=\mathrm{N}), 1504(\mathrm{C}=\mathrm{C}), 1254(\mathrm{C}-\mathrm{O}), 1046(\mathrm{C}-$ O), $838,774 \mathrm{~cm}^{-1}$.

Elemental Analysis calc. for $\mathrm{C}_{14} \mathrm{H}_{16} \mathrm{~N}_{2} \mathrm{OSi}$ : C 65.59, N, 10.93, H 6.29; found: C 64.85, N 10.66, H 5.98. 


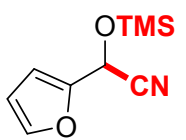

2j

2-(Furan-2-yl)-2-((trimethylsilyl)oxy)acetonitrile (2j). This product has been previously reported. ${ }^{13}$

${ }^{1}$ H NMR (300.13 MHz, CDCl 3 ): $\delta 7.45(\mathrm{~d}, J=1.7 \mathrm{~Hz}, 1 \mathrm{H}, \mathrm{CH}), 6.54(\mathrm{~d}, J=3.3 \mathrm{~Hz}, 1 \mathrm{H}, \mathrm{CH}), 6.40(\mathrm{dd}, J$ $=3.3,1.7 \mathrm{~Hz}, 1 \mathrm{H}, \mathrm{CH}), 5.54(\mathrm{~s}, 1 \mathrm{H}, \mathrm{CHCN}), 0.19(\mathrm{~s}, 9 \mathrm{H}, \mathrm{TMS}) \mathrm{ppm}$.

${ }^{13}$ CNMR (75.48 MHz, CDCl 3 ): $\delta 148.2$ (C), 143.8 (FurCH), 117.1 (CN), 110.8 (FurCH), 109.7 (FurCH), $57.4(\mathrm{CH}),-0.42$ (TMS) ppm.

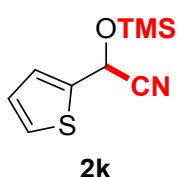

2-(Thiophen-2-yl)-2-((trimethylsilyl)oxy)acetonitrile (2k). This product has been previously reported. ${ }^{13}$ Error! Bookmark not defined.

${ }^{1} \mathbf{H}$ NMR (300.13 MHz, $\left.\mathbf{C D C l}_{3}\right): \delta 7.37$ (d, $\left.J=5.3 \mathrm{~Hz}, 1 \mathrm{H}, \mathrm{CH}\right), 7.20-7.15(\mathrm{~m}, 1 \mathrm{H}, \mathrm{CH}), 7.05-7.00(\mathrm{~m}$, $1 \mathrm{H}, \mathrm{CH}), 5.73$ (s, 1H, CHCN), 0.23 (s, 9H, TMS) ppm.

${ }^{13}$ C NMR (75.48 MHz, CDCl 3 ): $\delta 139.5$ (C), 127.2 (TiophCH), 126.9 (TiophCH), 126.3 (TiophCH), 118.3 (CN), $59.5(\mathrm{CH}),-0.31$ (TMS) ppm.

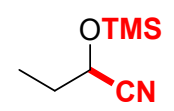

21

2-((Trimethylsilyl)oxy)butanenitrile (2I). This product has been previously reported. ${ }^{14}$

${ }^{1}$ H NMR (300.13 MHz, $\left.\mathbf{C D C l}_{3}\right): \delta 4.34(\mathrm{t}, J=6.3 \mathrm{~Hz}, 1 \mathrm{H}, \mathrm{CH}), 1.85-1.75(\mathrm{~m}, 2 \mathrm{H}, \mathrm{CH} 2), 1.04(\mathrm{t}, J=7.4$ $\mathrm{Hz}, 3 \mathrm{H}, \mathrm{CH} 3), 0.21$ (s, 9H, $\left.\mathrm{CH}_{3} \times 3\right)$ ppm.

${ }^{13} \mathrm{C}$ NMR (75.48 MHz, CDCl $): \delta 119.9(\mathrm{CN}), 62.7(\mathrm{CH}), 29.6\left(\mathrm{CH}_{2}\right), 8.9\left(\mathrm{CH}_{3}\right), 0.4(\mathrm{TMS}) \mathrm{ppm}$.

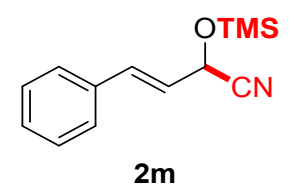

(E)-4-Phenyl-2-((trimethylsilyl)oxy)but-3-enenitrile (2m). This product has been previously reported. ${ }^{15}$

${ }^{1}$ H NMR (300.13 MHz, $\left.\mathbf{C D C l}_{3}\right)$ : $\delta 7.45-7.30(\mathrm{~m}, 5 \mathrm{H}, \mathrm{ArH}), 6.82(\mathrm{~d}, J=15.8 \mathrm{~Hz}, 1 \mathrm{H}, \mathrm{CH}), 6.20(\mathrm{dd}, J=$ $15.8,6.0 \mathrm{~Hz}, 1 \mathrm{H}, \mathrm{CH}), 5.13(\mathrm{~d}, J=6.0 \mathrm{~Hz}, 1 \mathrm{H}, \mathrm{CH}), 0.26$ (s, 9H, TMS) ppm. 
${ }^{13}$ C NMR (75.48 MHz, $\left.\mathbf{C D C l}_{3}\right): \delta 135.0\left(\mathrm{C}_{i p s o}\right) .133 .9(\mathrm{CH}), 128.76(\mathrm{ArCH}), 128.71(\mathrm{ArCH}), 127.0(\mathrm{ArCH})$, $123.5(\mathrm{CH}), 118.4(\mathrm{CN}), 62.2(\mathrm{CH}), 0.15(\mathrm{TMS}) \mathrm{ppm}$.

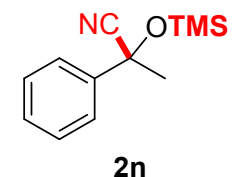

2-Phenyl-2-((trimethylsilyl)oxy)propanenitrile (2n). This product has been previously reported. ${ }^{16}$

${ }^{1} \mathbf{H}$ NMR (300.13 MHz, $\left.\mathbf{C D C l}_{3}\right): \delta$ 7.60-7.50 (m, 2H, ArH), 7.45-7.30 (m, 3H, ArH), $1.86\left(\mathrm{~s}, 3 \mathrm{H}, \mathrm{CH}_{3}\right)$, 0.18 (s, 9H, TMS) ppm.

${ }^{13} \mathrm{C}$ NMR (75.48 MHz, $\left.\mathbf{C D C l}_{3}\right): \delta 142.0\left(\mathrm{C}_{i p s o}\right), 128.68(\mathrm{ArCH}), 128.66(\mathrm{ArCH}), 124.6(\mathrm{ArCH}), 121.6$ $(\mathrm{CN}), 71.6(\mathrm{C}), 33.5\left(\mathrm{CH}_{3}\right), 1.03(\mathrm{TMS}) \mathrm{ppm}$.

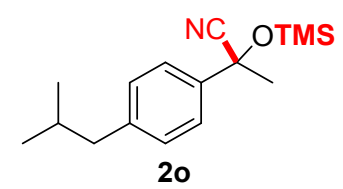

2-(4-Isobutylphenyl)-2-((trimethylsilyl)oxy)propanenitrile (20). This product has been previously reported. ${ }^{17}$

${ }^{1}$ H NMR (300.13 MHz, $\left.\mathbf{C D C l}_{3}\right): \delta 7.44(\mathrm{~d}, J=8.2 \mathrm{~Hz}, 2 \mathrm{H}, \mathrm{ArH}), 7.16(\mathrm{~d}, J=8.2 \mathrm{~Hz}, 2 \mathrm{H}, \mathrm{ArH}), 2.49(\mathrm{~d}$, $\left.J=7.2 \mathrm{~Hz}, 2 \mathrm{H}, \mathrm{CH}_{2}\right), 1.90-1.80\left(\mathrm{~m}, 4 \mathrm{H}, \mathrm{CH}, \mathrm{CH}_{3}\right), 0.90\left(\mathrm{~d}, J=6.6 \mathrm{~Hz}, 6 \mathrm{H}, 2 \times \mathrm{CH}_{3}\right), 0.16(\mathrm{~s}, 9 \mathrm{H}, \mathrm{TMS})$ ppm.

${ }^{13}$ C NMR (75.48 MHz, CDCl $\left._{3}\right): \delta 142.3\left(\mathrm{C}_{\text {ipso }}\right), 139.2\left(\mathrm{C}_{\text {ipso }}\right), 129.3(\mathrm{ArCH}), 124.5(\mathrm{ArCH}), 121.8(\mathrm{CN})$, $71.5(\mathrm{C}), 44.9\left(\mathrm{CH}_{2}\right), 33.4\left(\mathrm{CH}_{3}\right), 30.1\left(\mathrm{CH}_{3}\right), 22.3\left(\mathrm{CH}_{3}\right), 1.04$ (TMS) ppm.

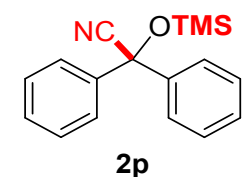

2,2-Diphenyl-2-((trimethylsilyl)oxy)acetonitrile (2p). This product has been previously reported. ${ }^{18}$

${ }^{1}$ H NMR (300.13 MHz, CDCl ${ }_{3}$ ): $\delta$ 7.55-7.50 (m, 4H, ArH), 7.40-7.35 (m, 6H, ArH), 0.15 (s, 9H, TMS) ppm.

${ }^{13}$ C NMR (75.48 MHz, CDCl $\left._{3}\right): \delta 141.9\left(\mathrm{C}_{i p s o}\right), 128.6(\mathrm{ArCH}), 128.5(\mathrm{ArCH}), 125.9(\mathrm{ArCH}), 120.7(\mathrm{CN})$, 76.3 (C), 0.9 (TMS) ppm.

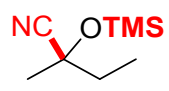

$2 q$

2-Methyl-2-((trimethylsilyl)oxy)butanenitrile (2q). This product has been previously reported. ${ }^{19}$ 
${ }^{1} \mathbf{H}$ NMR (300.13 MHz, CDCl $)$ : $\delta 1.85-1.65\left(\mathrm{~m}, 2 \mathrm{H}, \mathrm{CH}_{2}\right), 1.55\left(\mathrm{~s}, 3 \mathrm{H}, \mathrm{CH}_{3}\right), 1.04(\mathrm{t}, J=7.4 \mathrm{~Hz}, 3 \mathrm{H}$, $\left.\mathrm{CH}_{3} \mathrm{CH}_{2}\right), 0.23$ (s, 9H, TMS) ppm.

${ }^{13}$ C NMR (75.48 MHz, $\left.\mathbf{C D C l}_{3}\right): \delta 121.9(\mathrm{CN}), 70.2(\mathrm{C}), 36.4\left(\mathrm{CH}_{2}\right), 28.4\left(\mathrm{CH}_{3}\right), 8.6\left(\mathrm{CH}_{3}\right), 1.21(\mathrm{TMS})$ ppm.<smiles>N#CC1(O)CCCCC1</smiles>

$2 r$

1-((Trimethylsilyl)oxy)cyclohexane-1-carbonitrile (2r). This product has been previously reported. ${ }^{19}$

${ }^{1} \mathbf{H}$ NMR (300.13 MHz, $\left.\mathbf{C D C l}_{3}\right): \delta 2.10-2.00\left(\mathrm{~m}, 2 \mathrm{H}, \mathrm{CH}_{2}\right), 1.75-1.70\left(\mathrm{~m}, 2 \mathrm{H}, \mathrm{CH}_{2}\right), 1.70-1.45(\mathrm{~m}, 6 \mathrm{H}$, $\mathrm{CH}_{2}$ x 3), 1.30-1.20 (m, 2H, $\left.\mathrm{CH}_{2}\right), 0.23$ (s, 9H, TMS) ppm.

${ }^{13}$ C NMR (75.48 MHz, $\left.\mathbf{C D C l}_{3}\right): \delta 121.9(\mathrm{CN}), 70.6(\mathrm{C}), 39.3\left(\mathrm{CH}_{2}\right), 24.5\left(\mathrm{CH}_{2}\right), 22.6\left(\mathrm{CH}_{2}\right), 1.38(\mathrm{TMS})$ ppm.<smiles>COC1(C)CCCc2ccccc21</smiles>

1-((Trimethylsilyl)oxy)-1,2,3,4-tetrahydronaphthalene-1-carbonitrile (2s). This product has been previously reported. ${ }^{20}$

${ }^{1} \mathbf{H}$ NMR (300.13 MHz, $\left.\mathbf{C D C l}_{3}\right): \delta$ 7.70-7.60 (m, 1H, ArH), 7.30-7.20 (m, 2H, ArH), 7.15-7.05 (m, 1H, ArH), 2.85-2.80 (m, 2H), 2.40-2.30 (m, 1H), 2.25-2.15 (m, 1H), 2.10-1.95 (m, 2H), 0.21 (s, 9H, TMS) ppm.

${ }^{13} \mathrm{C} \mathrm{NMR}\left(75.48 \mathrm{MHz}, \mathrm{CDCl}_{3}\right): \delta 136.1\left(\mathrm{C}_{\text {ipso }}\right), 135.7\left(\mathrm{C}_{\text {ipso }}\right), 129.3(\mathrm{ArCH}), 129.1(\mathrm{ArCH}), 128.0(\mathrm{ArCH})$, 126.6 (ArCH), $122.1(\mathrm{CN}), 69.6(\mathrm{C}), 37.7\left(\mathrm{CH}_{2}\right), 28.3\left(\mathrm{CH}_{2}\right), 18.7\left(\mathrm{CH}_{2}\right), 1.3(\mathrm{TMS}) \mathrm{ppm}$.

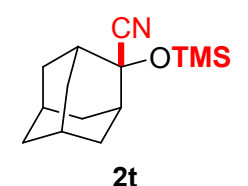

2-((Trimethylsilyl)oxy)adamantane-2-carbonitrile (2t). This product has been previously reported. ${ }^{21,22}$

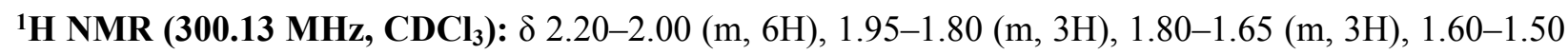
(m, 2H), 0.25 (s, 9H, TMS) ppm.

${ }^{13}$ C NMR (75.48 MHz, $\left.\mathbf{C D C l}_{3}\right): \delta 122.1(\mathrm{CN}), 74.7(\mathrm{C}), 38.1(\mathrm{CH}), 37.2\left(\mathrm{CH}_{2}\right), 34.7\left(\mathrm{CH}_{2}\right), 30.9\left(\mathrm{CH}_{2}\right)$, $26.3(\mathrm{CH}), 26.1(\mathrm{CH}), 1.2$ (TMS) ppm. 


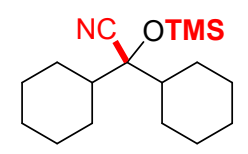

$2 \mathbf{u}$

2,2-Dicyclohexyl-2-((trimethylsilyl)oxy)acetonitrile (2u).

${ }^{1}$ H NMR (300.13 MHz, CDCl 3 ): $\delta 1.90-1.75(\mathrm{~m}, 8 \mathrm{H}), 1.70-1.60(\mathrm{~m}, 4 \mathrm{H}), 1.30-1.05\left(\mathrm{~m}, 10 \mathrm{H}, \mathrm{CH}_{2}\right), 0.23$ (s, 9H, TMS) ppm.

${ }^{13} \mathrm{C}$ NMR (75.48 MHz, $\left.\mathbf{C D C l}_{3}\right): \delta 120.5(\mathrm{CN}), 80.6(\mathrm{C}), 43.6(\mathrm{CH}), 28.3\left(\mathrm{CH}_{2}\right), 26.4\left(\mathrm{CH}_{2}\right), 26.2\left(\mathrm{CH}_{2}\right)$, $26.1\left(\mathrm{CH}_{2}\right), 1.9$ (TMS) ppm.

IR (ATR): v $2931\left(\mathrm{CH}_{2}\right), 2858\left(\mathrm{CH}_{2}\right), 1451,1249,1123,1023,842,766 \mathrm{~cm}^{-1}$.

Elemental Analysis calc. for $\mathrm{C}_{17} \mathrm{H}_{31} \mathrm{NOSi}$ C 69.56, N 4.77, H 10.65; found: C 69.62, N 4.68, H 10.21. 
14. Recyclability of the reaction.

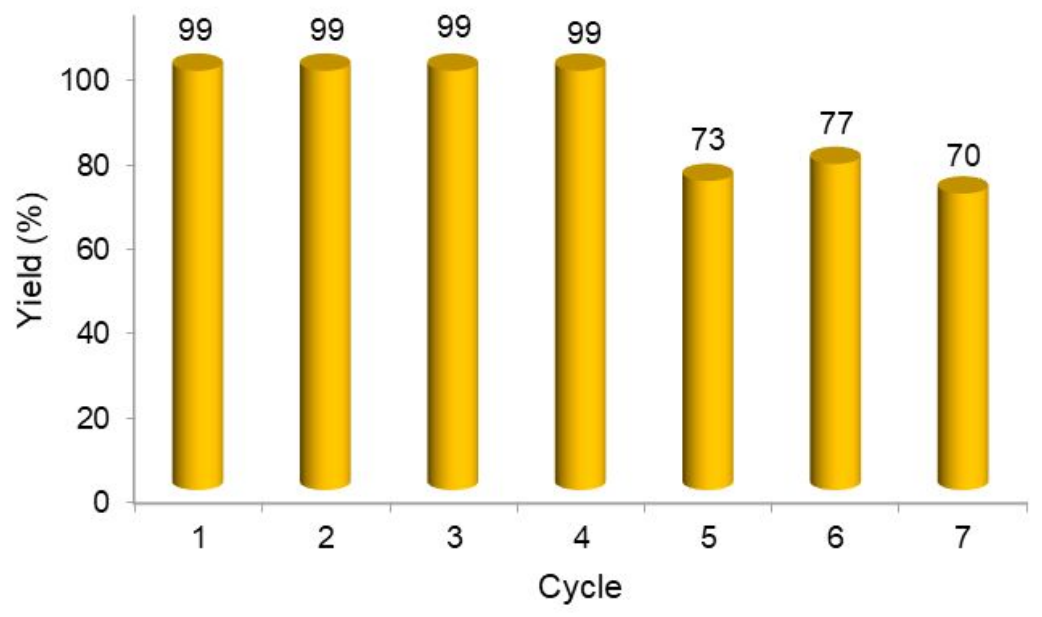

Figure S10. Study of the recyclability of catalyst GR-MOF-6 under solvent-free optimized conditions.

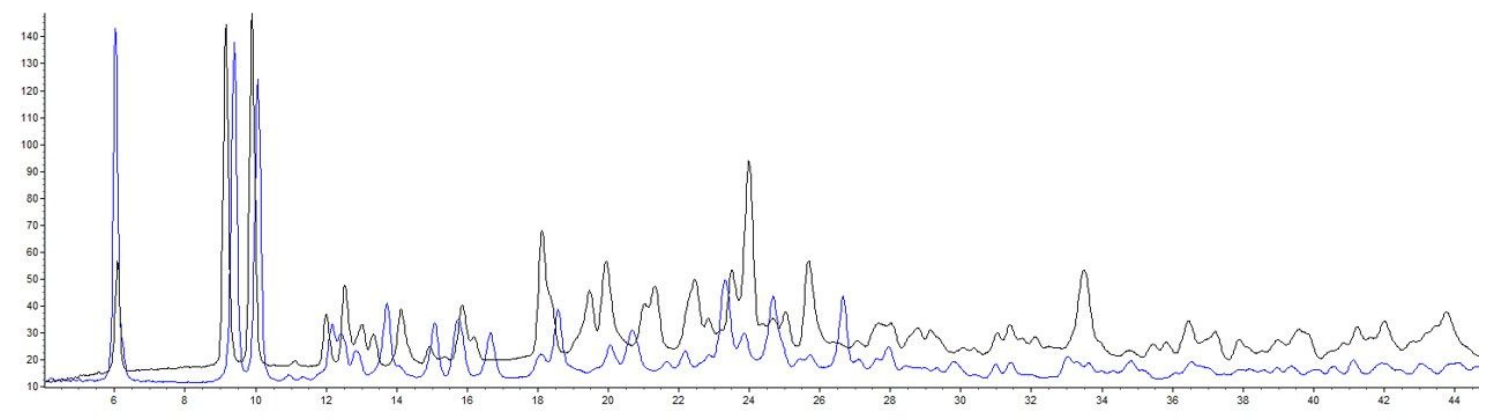

Figure S11. Powder XR patterns showing the recyclability of catalyst. XRP of initial GR-MOF-6 (black) and after the fifth cycle (blue). 
15. Studies of the mechanism of the reaction using IR (ATR).

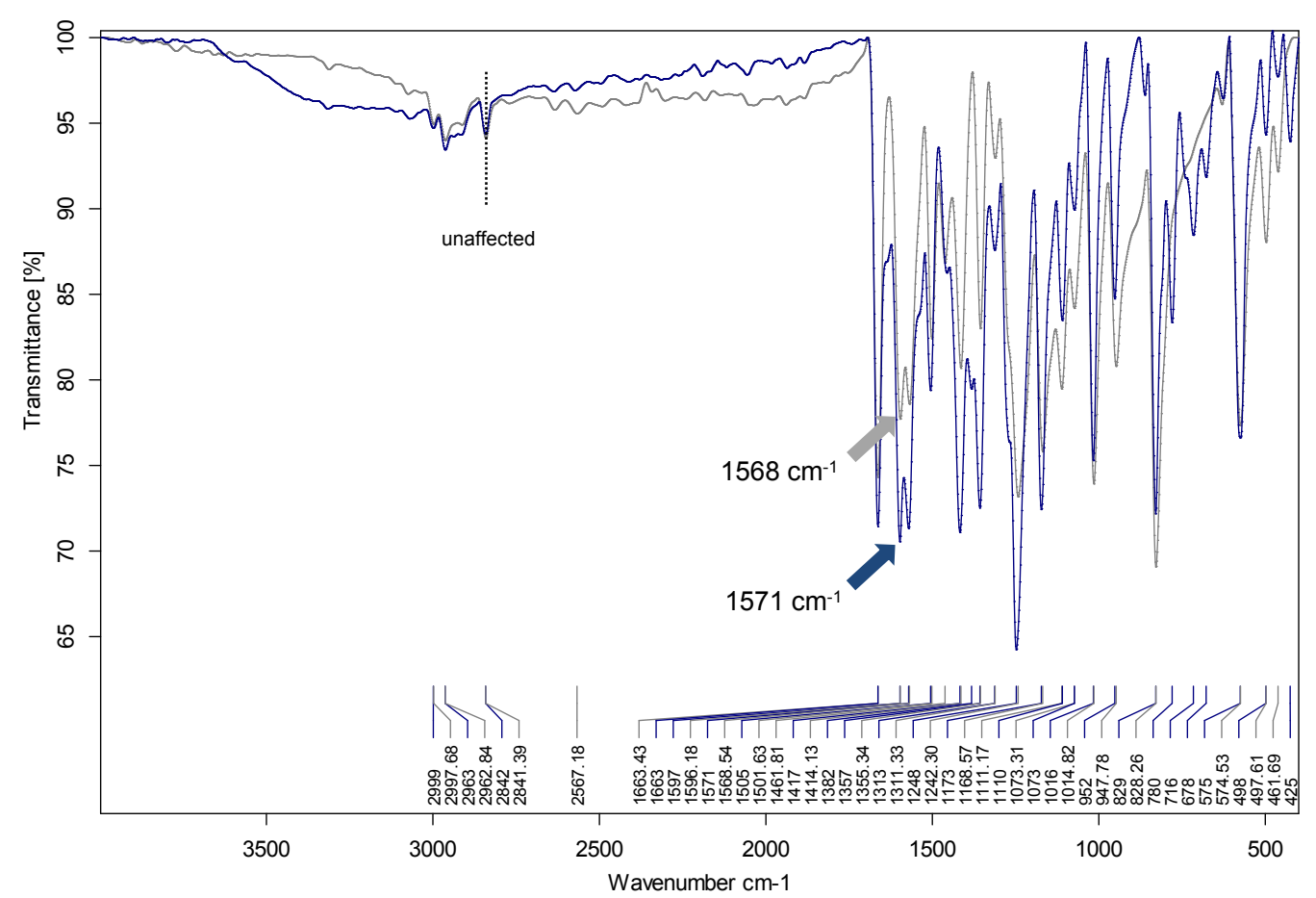

Figure S12. IR(ATR) spectra of 1-(4-methoxyphenyl)ethan-1-one (grey) and the mixture of GRMOF-6 and 1-(4-methoxyphenyl)ethan-1-one in a ratio 1:1 (blue).

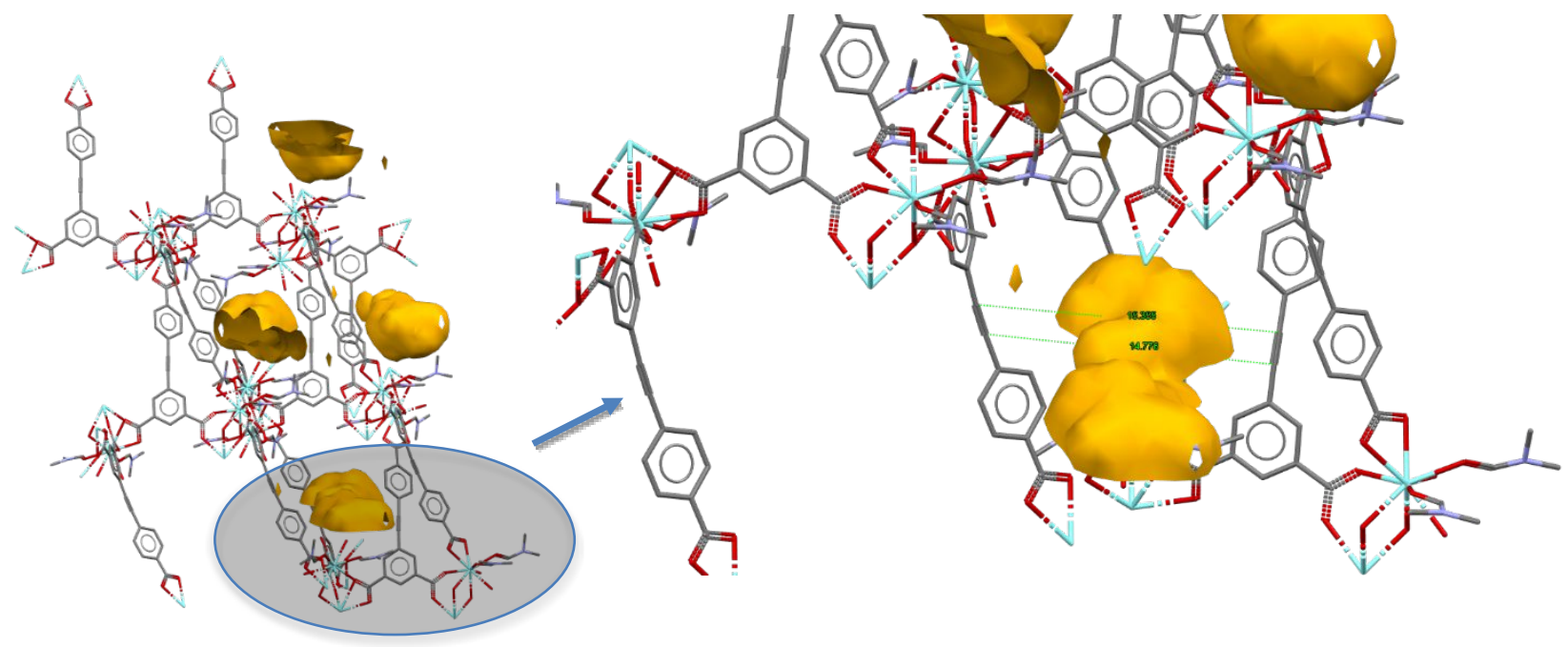

Figure S13. Views of the packing showing the solvent-accessible voids. 


\section{Previous results obtained with different lanthanides MOF catalysts}

Table S5. Catalytic cyanosilylation of benzaldehyde performances of Ln-MOFs reported in the literature.

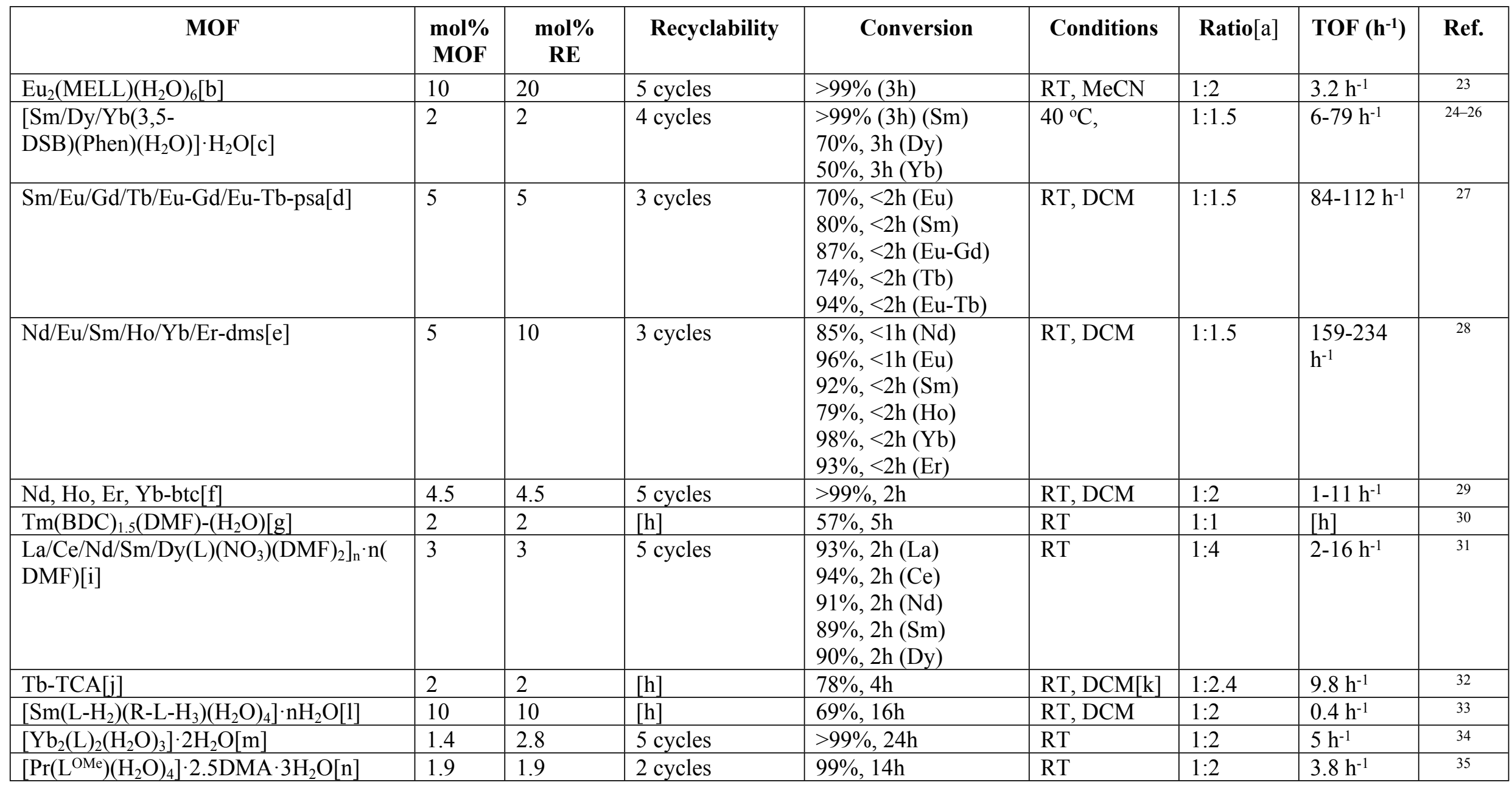




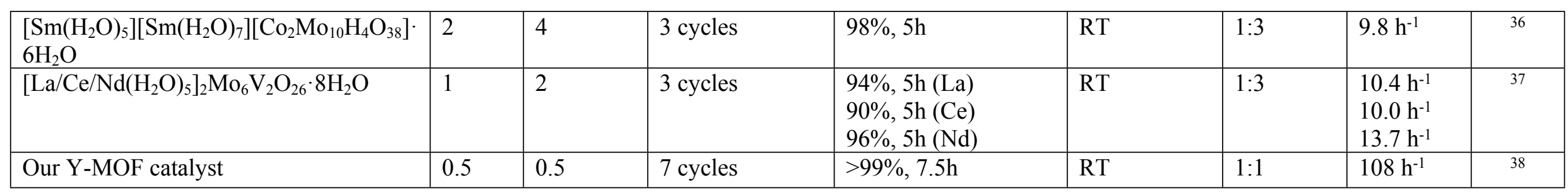

[a] Ratio between benzaldehyde and TMSCN; [b] MELL = mellitic acid; [c] 3,5-DSB = 3,5-disulfobenzoate, Phen = 1,10-phenanthroline; [d] psa = 2phenylsuccinate; [e] dms = 2,3-dimethylsuccinate; [f] btc = 1,3,5-benzenetricarboxylate; [g] BDC = 1,4-benzenedicarboxylate; [h] Not given; [i] L = 5-[2- \{2,4,6trioxotetrahydropyrimidin-5(2H)-ylidene\}hydrazinyl]isophthalate; [j] TCA = tricarboxytriphenylamine; [k] The aldehyde employed is 2-nitrobenzaldehyde. [1] L-H $\mathrm{H}_{4}=$ 2,2'-diethoxy-1,1'-binaphthalene-6,6'-bisphosphonic acid. [m] L = 4,4',4'-((2,4,6-trimethylbenzene-1,3,5-triyl)tris(methylene))tribenzoate; [n] L LMe $^{\prime}, 3^{\prime}$ $((2,3,6,7$-tetramethoxyanthracene-9,10-diyl)bis(4,1-phenylene) $)$ diacrylate. 


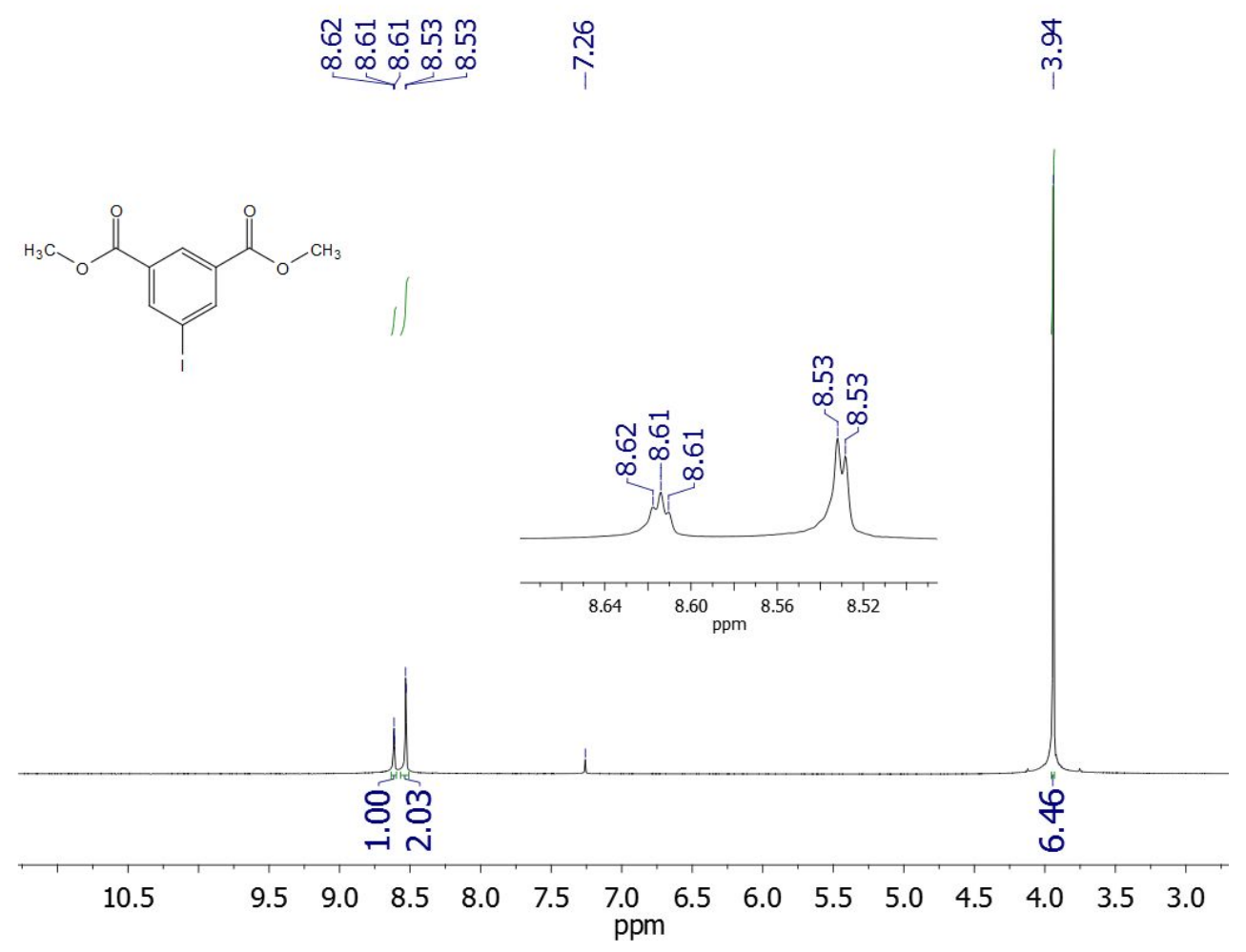

Figure S14. ${ }^{1} \mathrm{H}$ NMR (400 MHz, $\left.\mathrm{CDCl}_{3}, 298 \mathrm{~K}\right)$ spectrum of compound 4.
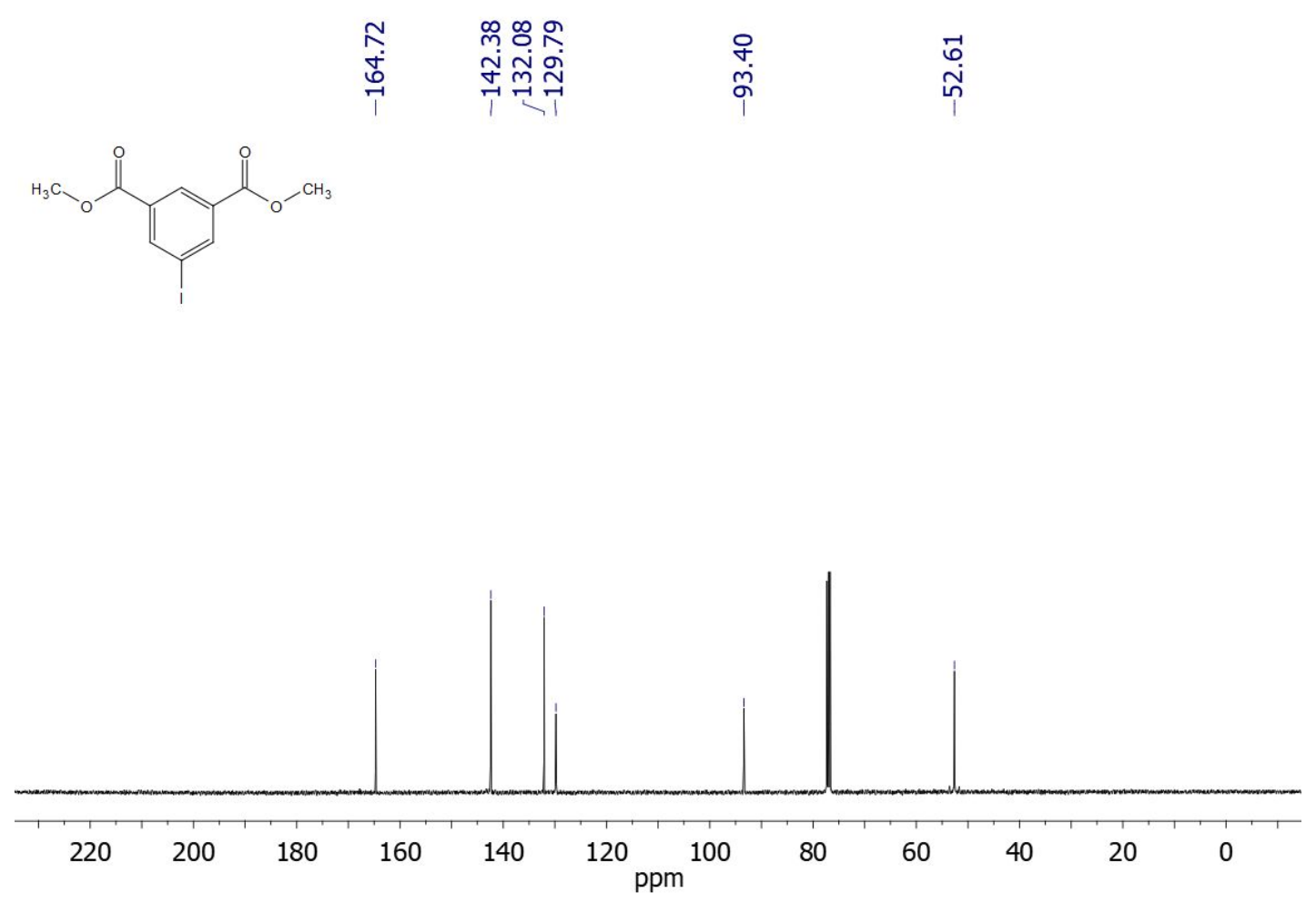

Figure S15. ${ }^{13} \mathrm{C}$ NMR $\left(100 \mathrm{MHz}, \mathrm{CDCl}_{3}, 298 \mathrm{~K}\right)$ spectrum of compound 4. 


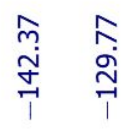

ํำ
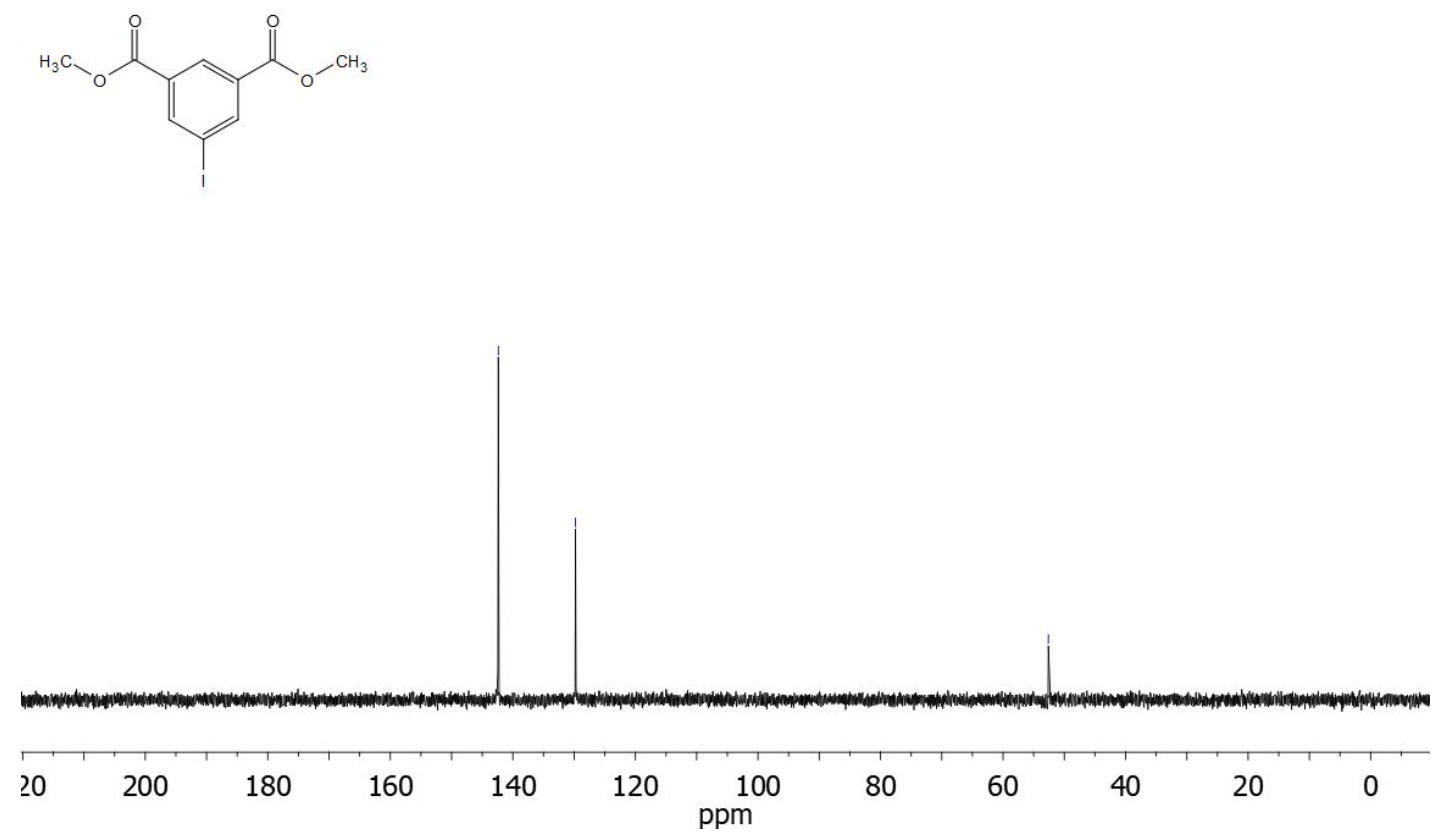

Figure S16. DEPT135 (100 MHz, CDCl 3 , 298K) spectrum of compound 4.

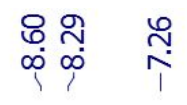

ঙ

ָָֻ

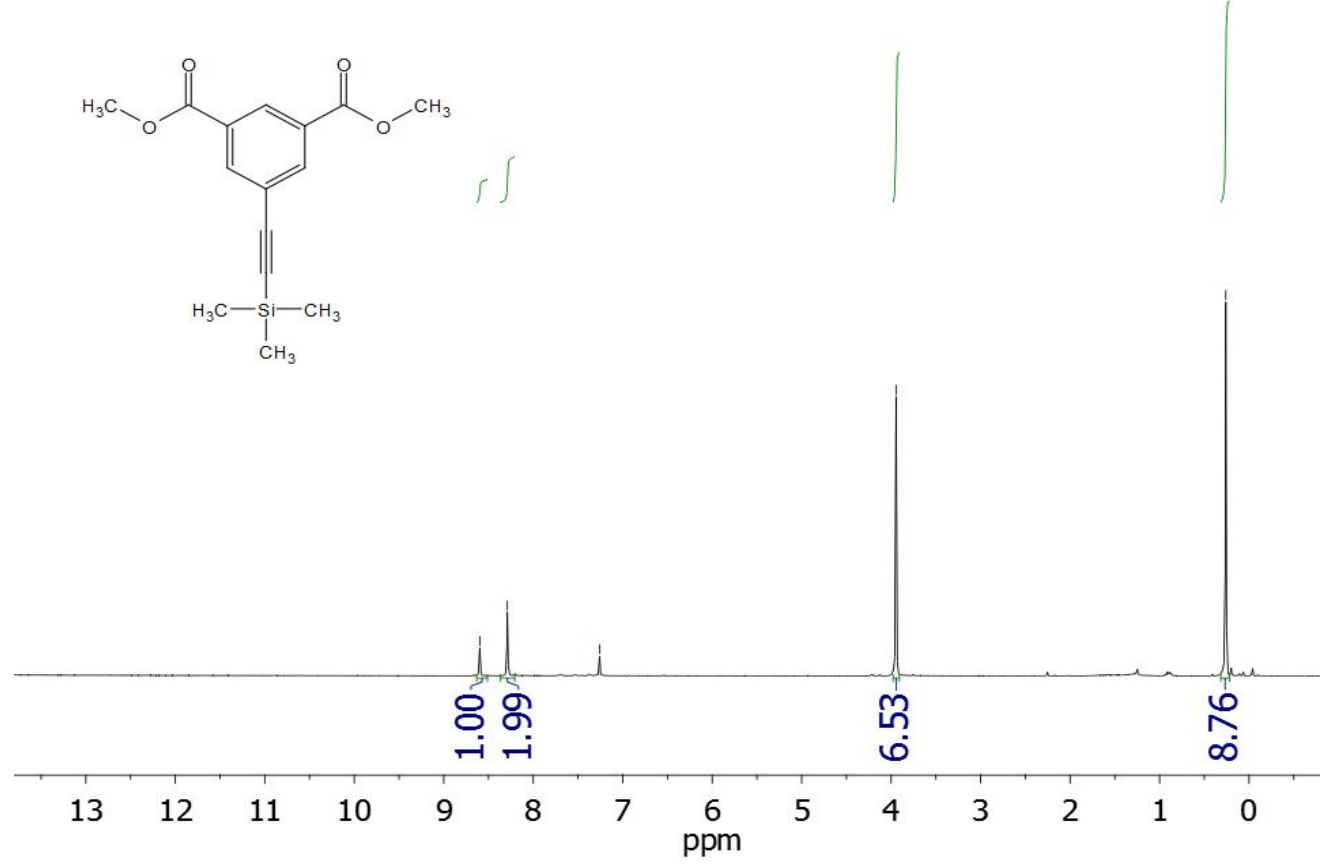

Figure S17. ${ }^{1} \mathrm{H}$ NMR (400 MHz, $\left.\mathrm{CDCl}_{3}, 298 \mathrm{~K}\right)$ spectrum of compound 5.

S30 


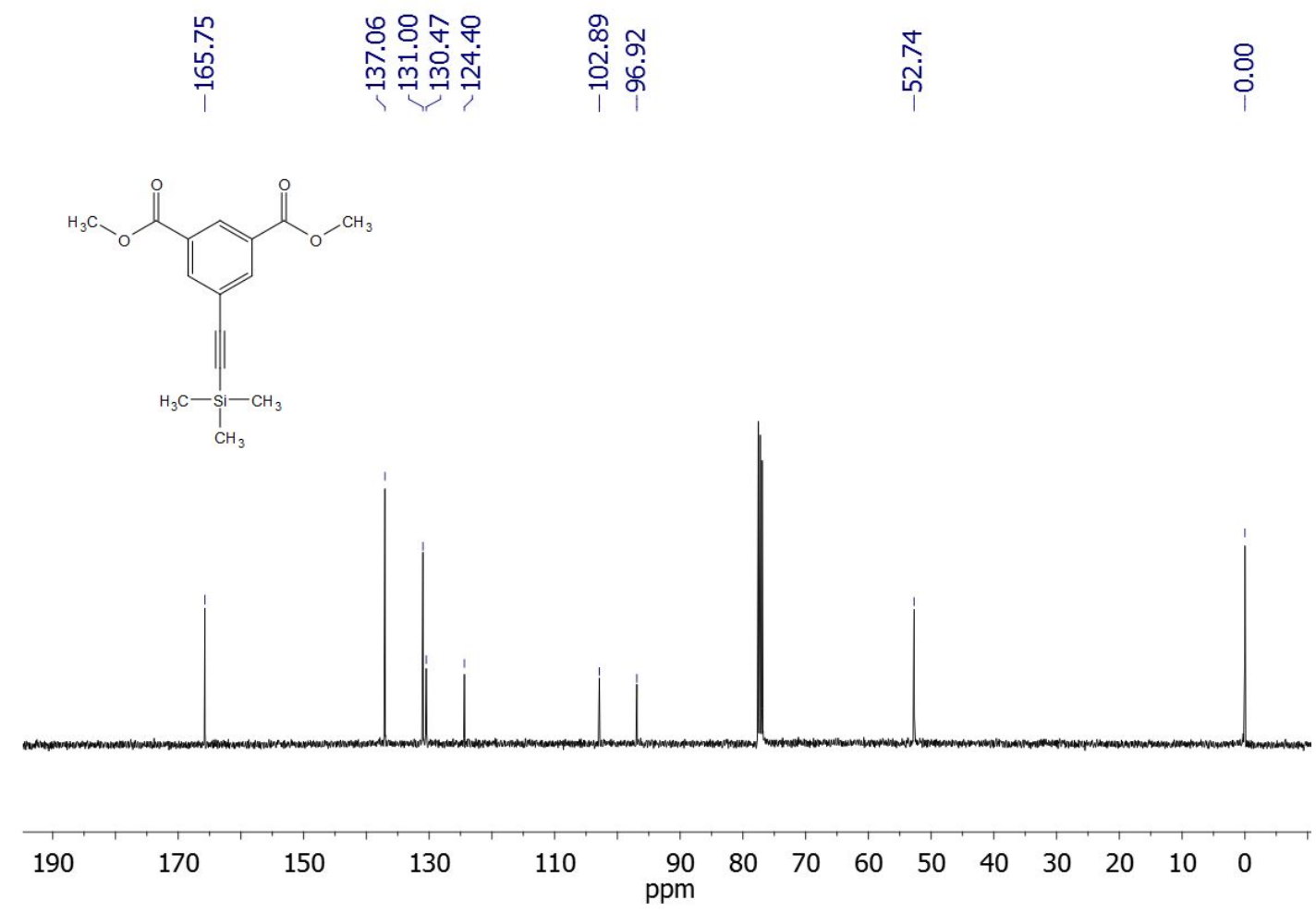

Figure S18. ${ }^{13} \mathrm{C}$ NMR $\left(100 \mathrm{MHz}, \mathrm{CDCl}_{3}, 298 \mathrm{~K}\right)$ spectrum of compound 5.

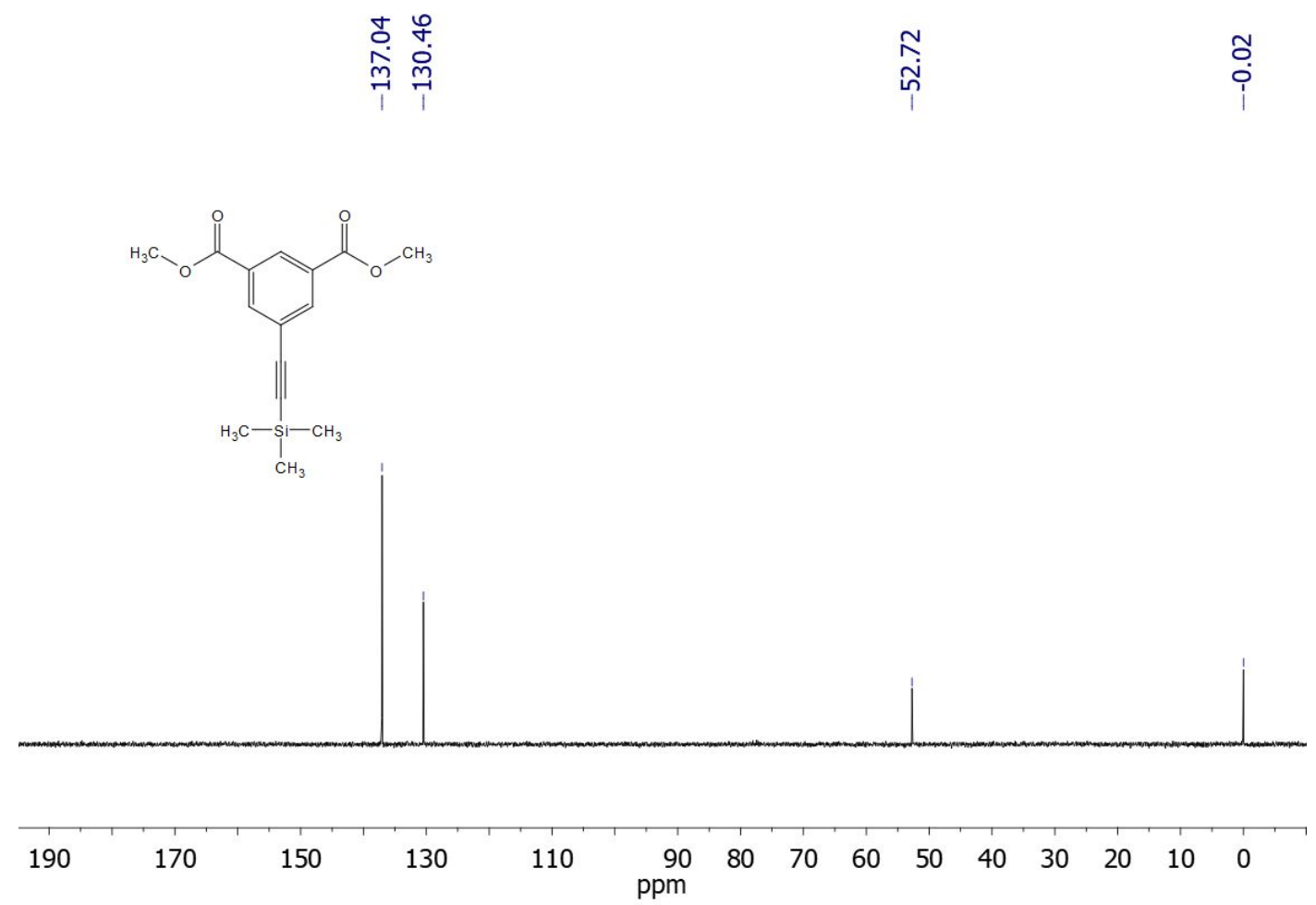

Figure S19. DEPT135 (100 MHz, $\left.\mathrm{CDCl}_{3}, 298 \mathrm{~K}\right)$ spectrum of compound $\mathbf{5}$. 


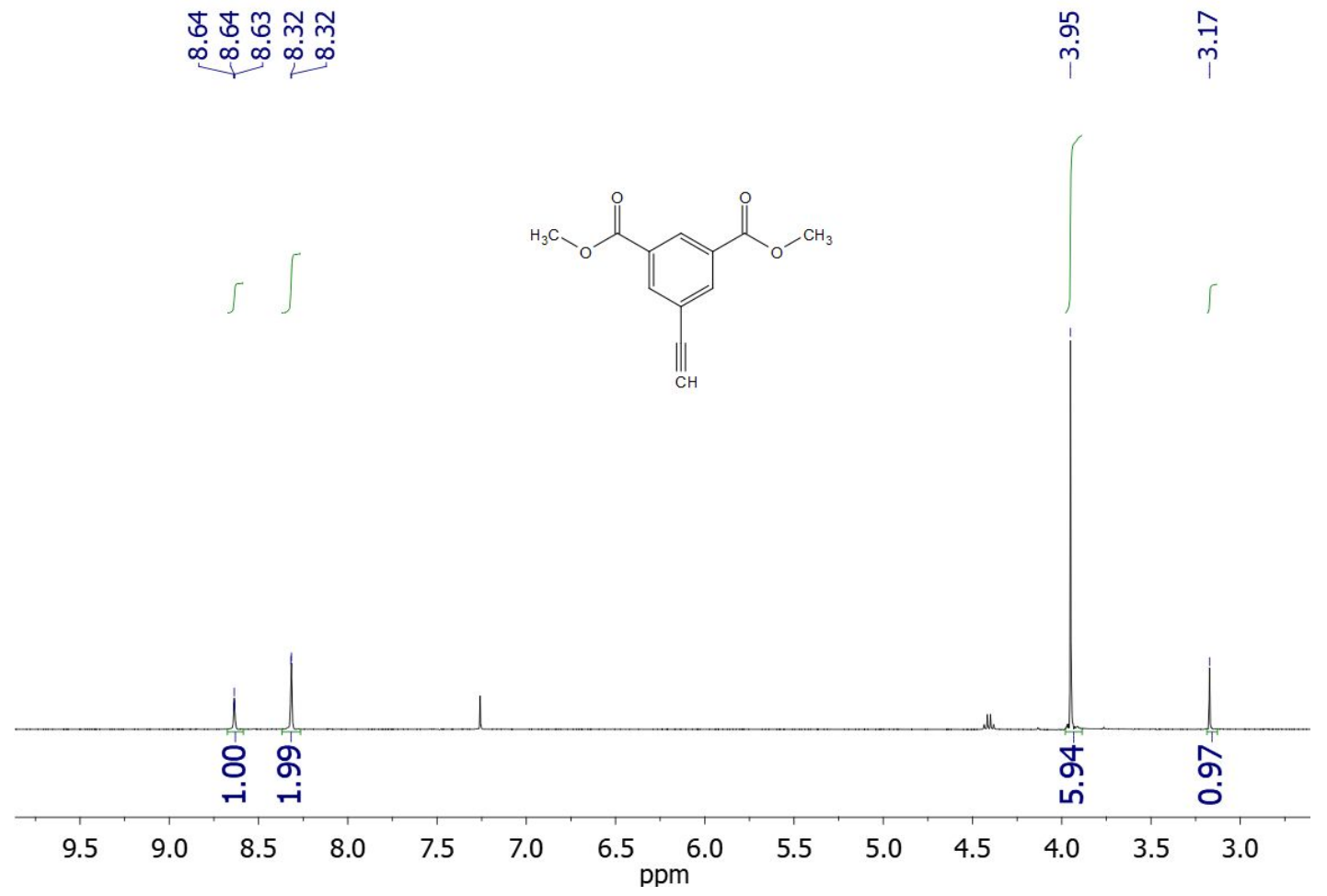

Figure S20. ${ }^{1} \mathrm{H}$ NMR (400 MHz, $\left.\mathrm{CDCl}_{3}, 298 \mathrm{~K}\right)$ spectrum of compound 6.
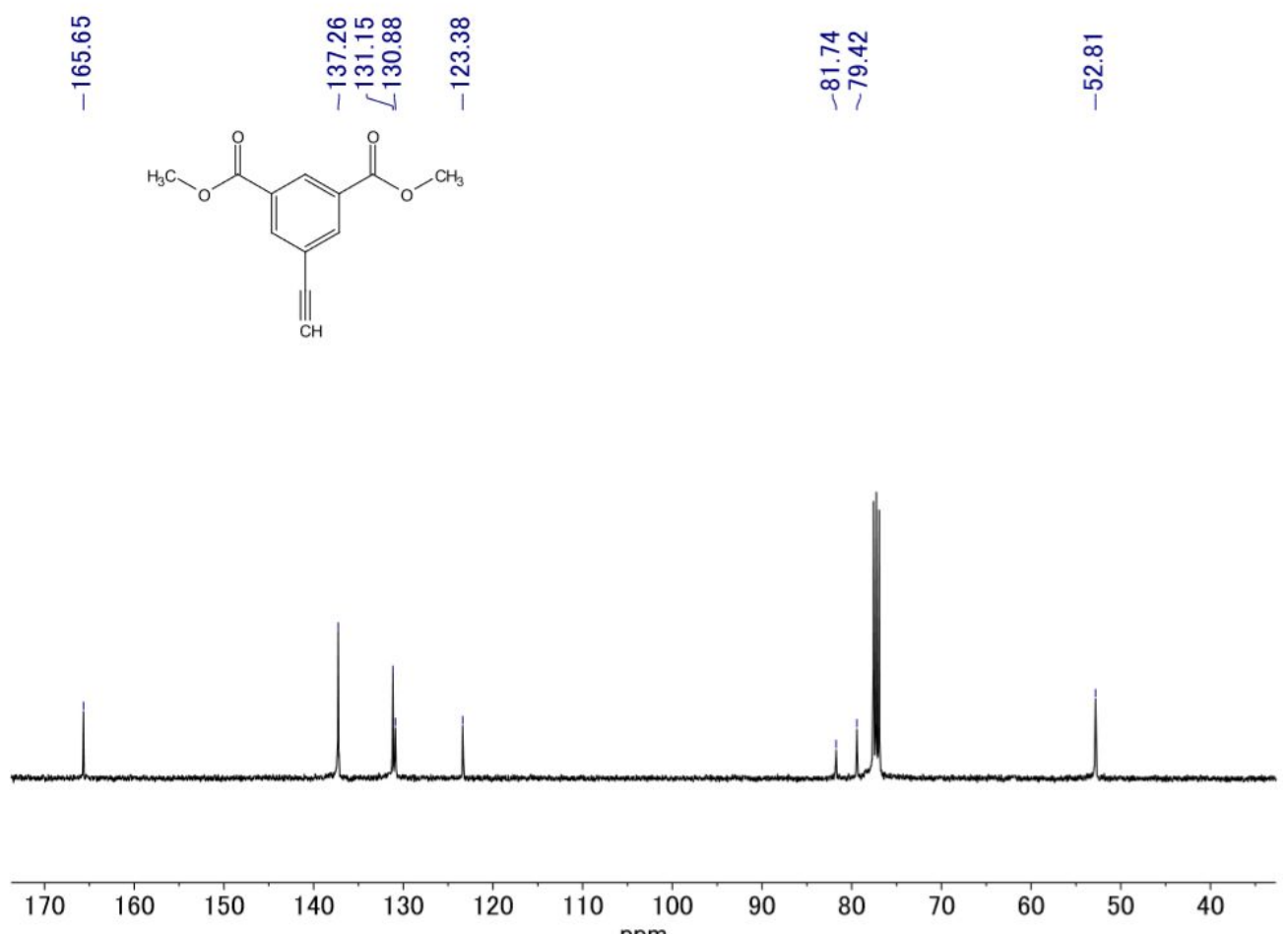

Figure S21. ${ }^{13} \mathrm{C}$ NMR $\left(100 \mathrm{MHz}, \mathrm{CDCl}_{3}, 298 \mathrm{~K}\right)$ spectrum of compound 6. 


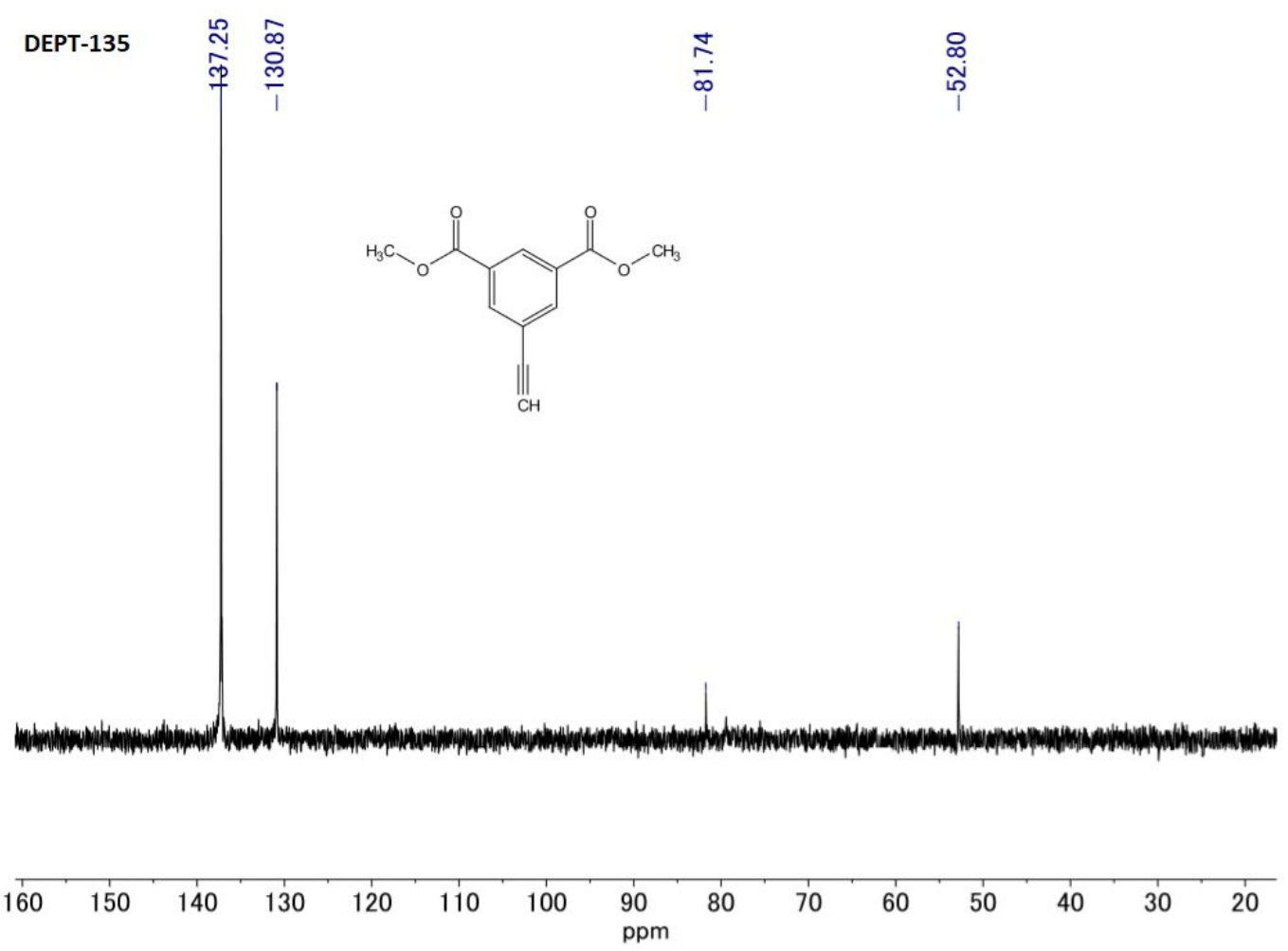

Figure S22. DEPT135 (100MHz, $\left.\mathrm{CDCl}_{3}, 298 \mathrm{~K}\right)$ spectrum of compound $\mathbf{6}$.

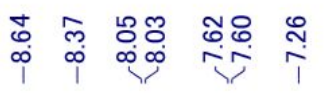
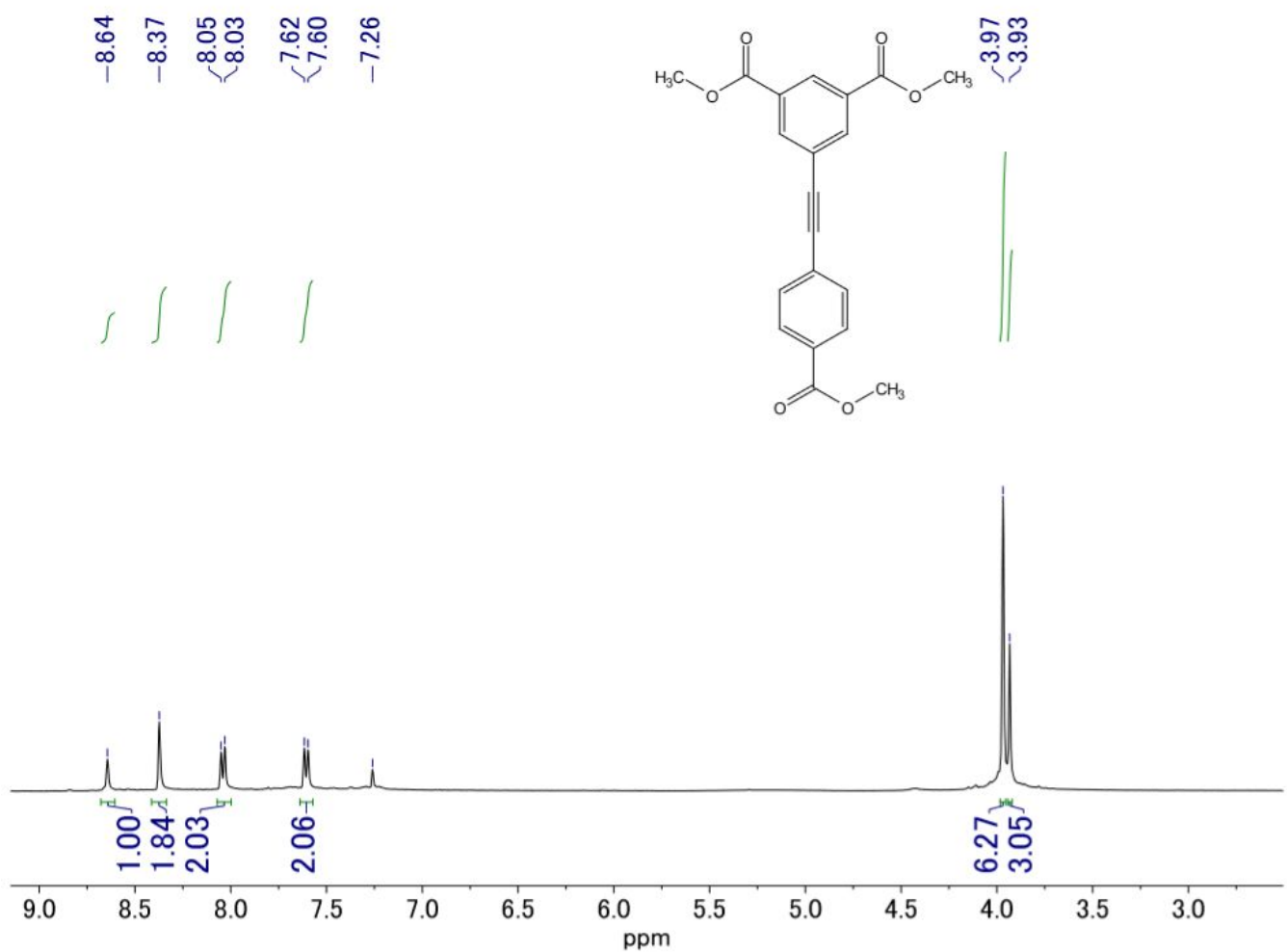

Figure S23. ${ }^{1} \mathrm{H}$ NMR $\left(400 \mathrm{MHz}, \mathrm{CDCl}_{3}, 298 \mathrm{~K}\right)$ spectrum of compound $\mathbf{8}$. 


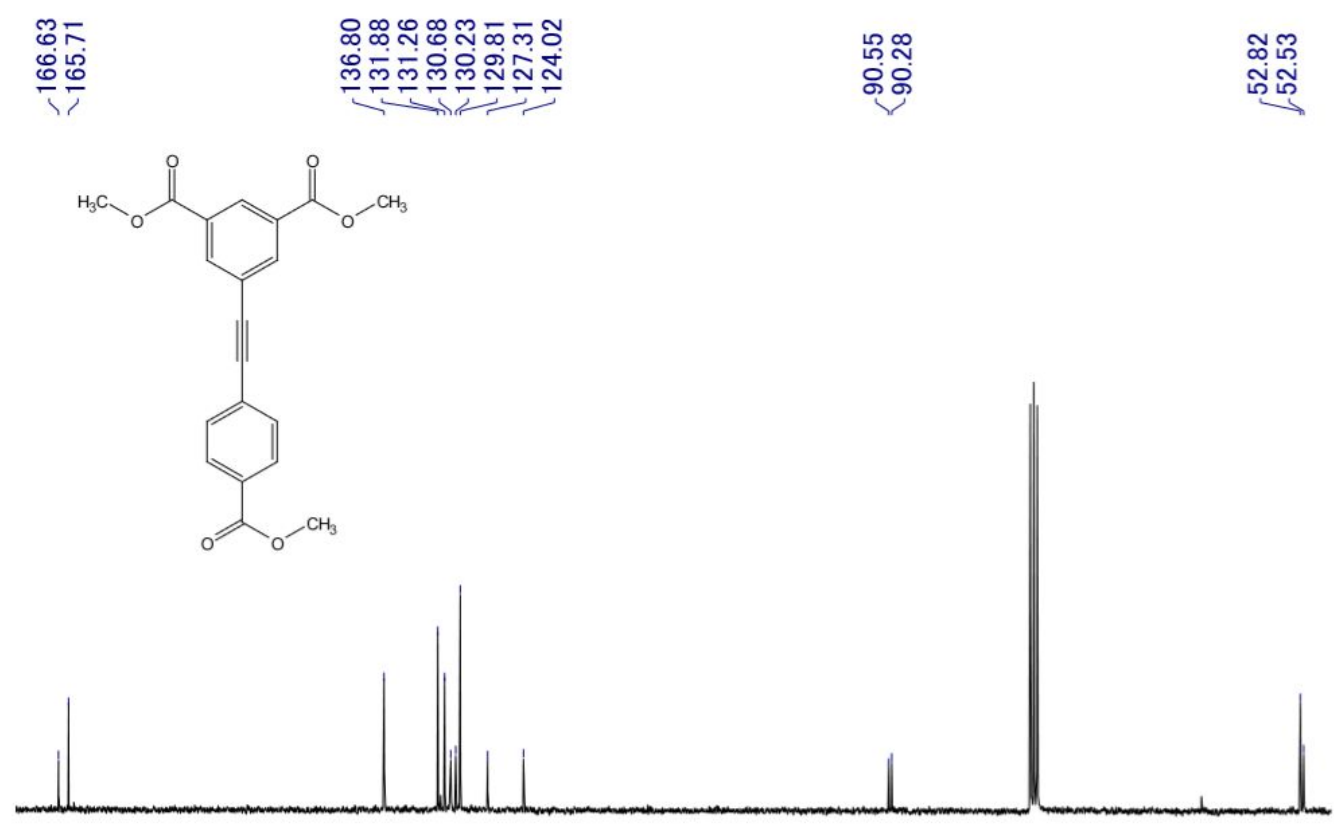

$\begin{array}{llllllllllllllllllllllllllllllll}170 & 165 & 160 & 155 & 150 & 145 & 140 & 135 & 130 & 125 & 120 & 115 & 110 & 105 & 100 & 95 & 90 & 85 & 80 & 75 & 70 & 65 & 60 & 55 & 50\end{array}$

Figure S24. ${ }^{13} \mathrm{C}$ NMR $\left(100 \mathrm{MHz}, \mathrm{CDCl}_{3}, 298 \mathrm{~K}\right)$ spectrum of compound $\mathbf{8}$.

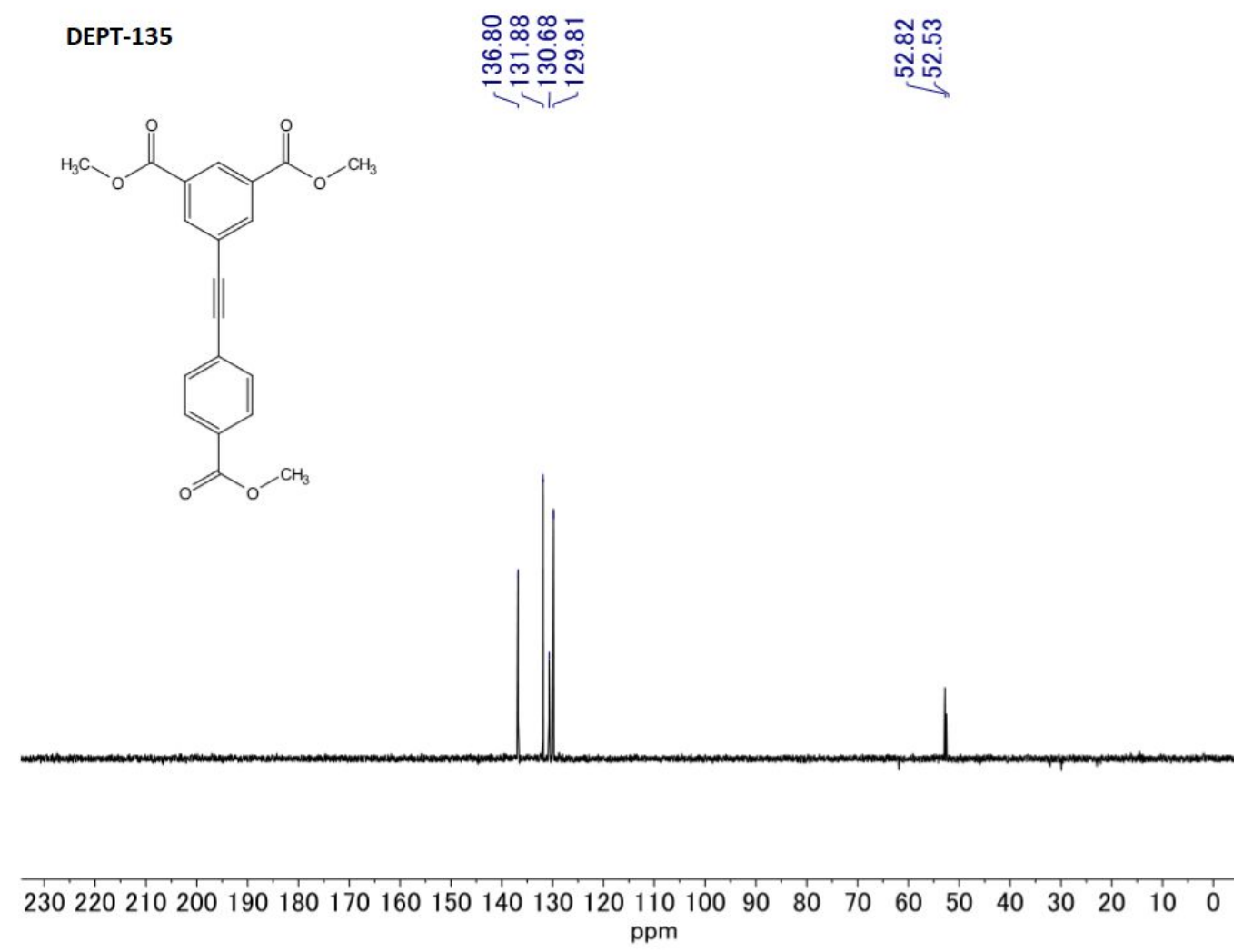

Figure S25. DEPT135 (100 MHz, CDCl, $298 \mathrm{~K})$ spectrum of compound 8. 


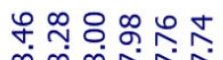

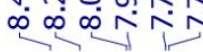

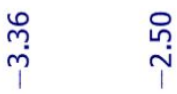
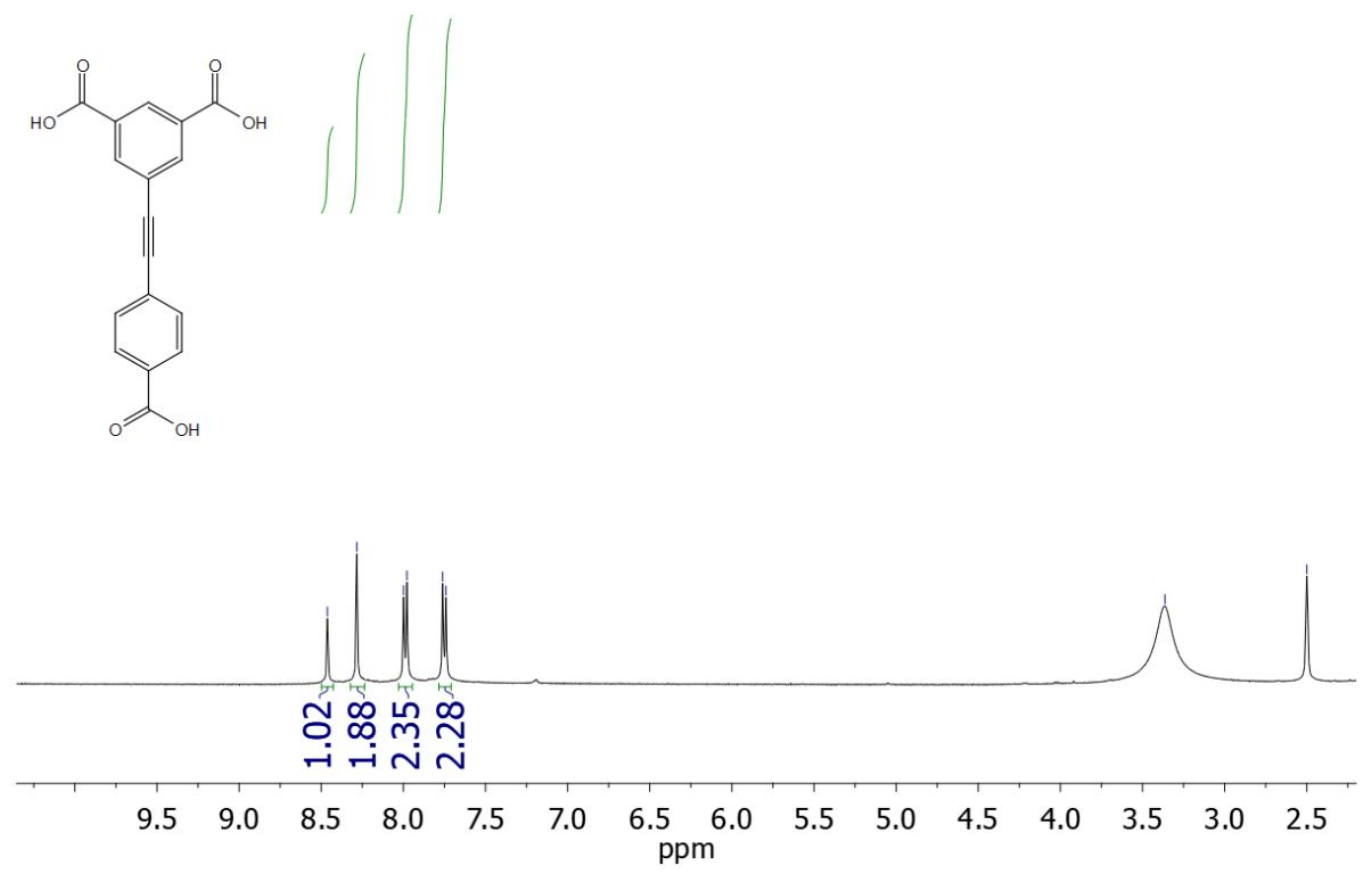

Figure S26. ${ }^{1} \mathrm{H}$ NMR (400 MHz, $\left.\mathrm{CDCl}_{3}, 298 \mathrm{~K}\right)$ spectrum of ligand $\mathbf{L}$.

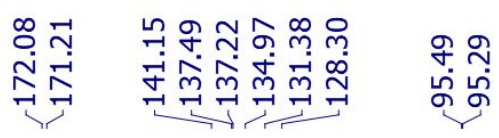<smiles>O=C(O)c1ccc(C#Cc2cc(C(=O)O)cc(C(=O)O)c2)cc1</smiles>

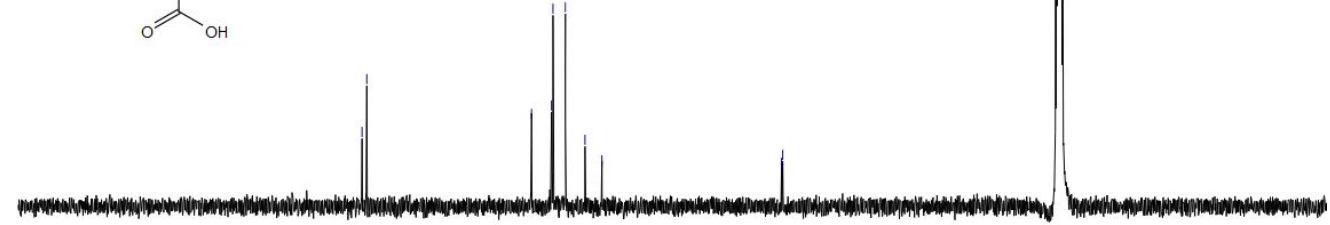

60

$40 \quad 20 \quad 0$

Figure S27. ${ }^{13} \mathrm{C}$ NMR $\left(100 \mathrm{MHz}, \mathrm{CDCl}_{3}, 298 \mathrm{~K}\right)$ spectrum of ligand $\mathbf{L}$. 


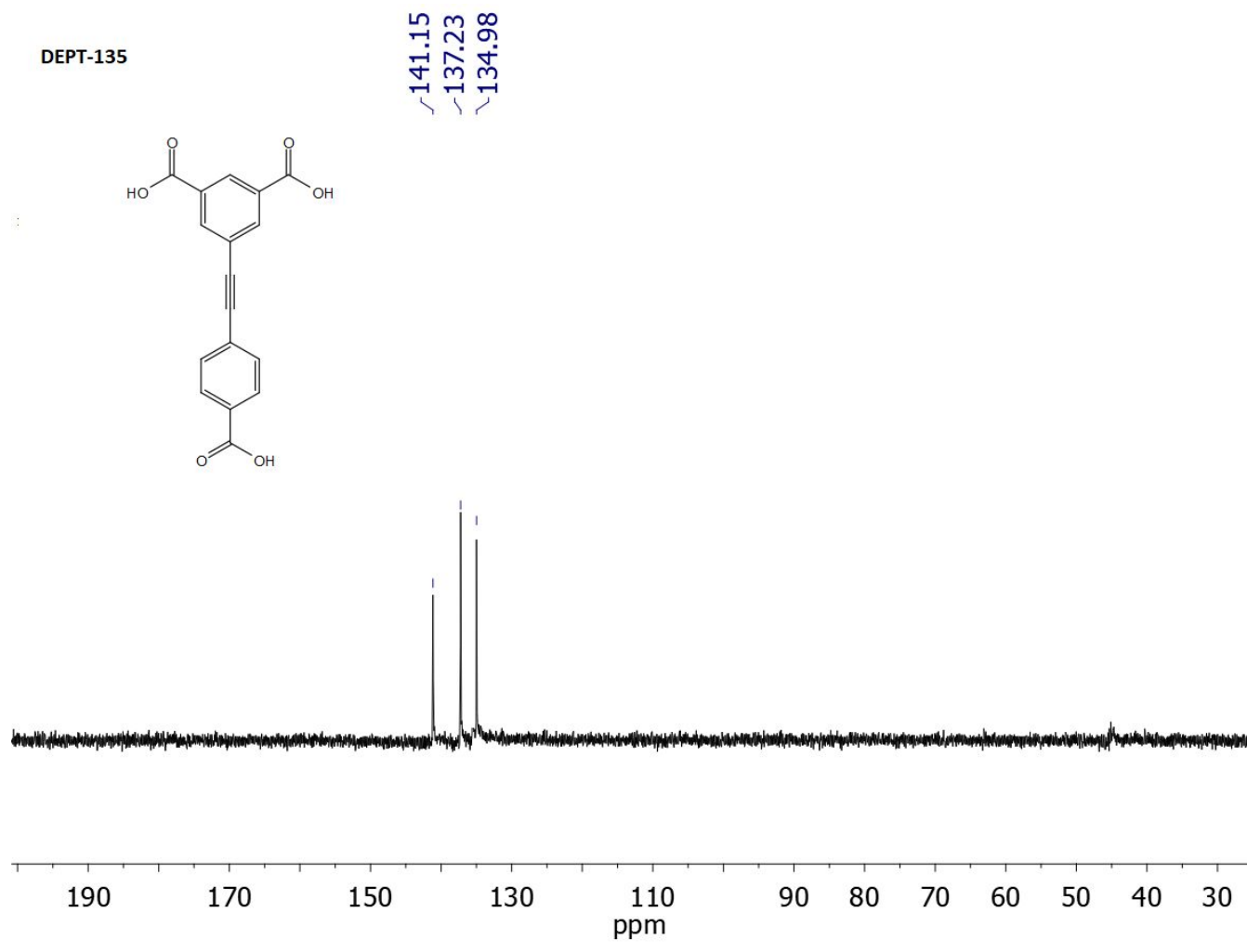

Figure S28. DEPT135 (100 MHz, $\left.\mathrm{CDCl}_{3}, 298 \mathrm{~K}\right)$ spectrum of ligand $\mathbf{L}$. 


\section{References}

(1) Bruker Apex2, B. A. I. Bruker Apex2, Bruker AXS Inc. Madison, Wisconsin, USA 2004.

(2) Sheldrick, G. M. SADABS. University of Gottingen: Gottingen, Germany 1996, p Program for Empirical Adsorption Correction.

(3) Sheldrick, G. M. A Short History of SHELX. Acta Crystallogr. Sect. A Found. Crystallogr. 2008, 64 (1), 112-122. https://doi.org/10.1107/S0108767307043930.

(4) Sheldrick, G. M. Crystal Structure Refinement with SHELXL. Acta Crystallogr. Sect. C Struct. Chem. 2015, 71, 3-8. https://doi.org/10.1107/S2053229614024218.

(5) Farrugia, L. J. WinGX and ORTEP for Windows: An Update. J. Appl. Crystallogr. 2012, 45 (4), 849-854. https://doi.org/10.1107/S0021889812029111.

(6) J. Rodríguez-Carvajal. FULLPROF 2000,Version 2.5d,. FULLPROF 2000,version 2.5d, Laboratoire Léon Brillouin (CEA-CNRS), Centre d'Études de Saclay, Gif sur Yvette Cedex, France,. 2000.

(7) Thavornpradit, S.; Killough, J. M.; Bergbreiter, D. E. Minimizing Solvent Waste in Catalytic Reactions in Highly Recyclable Hydrocarbon Solvents. Org. Biomol. Chem. 2020, 18 (22), 4248-4256. https://doi.org/10.1039/d0ob00734j.

(8) Zhu, C.; Tang, H.; Yang, K.; Wu, X.; Luo, Y.; Wang, J.; Li, Y. A Urea-Containing MetalOrganic Framework as a Multifunctional Heterogeneous Hydrogen Bond-Donating $\begin{array}{lllll}\text { Catalyst. Catal. } & \text { Commun. } & \text { 2020, } & 135, & 105837 .\end{array}$ https://doi.org/10.1016/j.catcom.2019.105837.

(9) Wang, W.; Luo, M.; Yao, W.; Ma, M.; Pullarkat, S. A.; Xu, L.; Leung, P. H. Catalyst-Free and Solvent-Free Cyanosilylation and Knoevenagel Condensation of Aldehydes. ACS Sustain. Chem. Eng. 2019, 7 (1), 1718-1722. https://doi.org/10.1021/acssuschemeng.8b05486.

(10) North, M.; Omedes-Pujol, M.; Young, C. Kinetics and Mechanism of the Racemic Addition of Trimethylsilyl Cyanide to Aldehydes Catalysed by Lewis Bases. Org. Biomol. Chem. 2012, 10 (21), 4289-4298. https://doi.org/10.1039/c2ob25188d.

(11) Gu, J. Z.; Wan, S. M.; Kirillova, M. V.; Kirillov, A. M. H-Bonded and Metal(Ii)-Organic Architectures Assembled from an Unexplored Aromatic Tricarboxylic Acid: Structural Variety and Functional Properties. Dalt. Trans. 2020, 49 (21), 7197-7209. https://doi.org/10.1039/d0dt01261k.

(12) Wu, W. B.; Zeng, X. P.; Zhou, J. Carbonyl-Stabilized Phosphorus Ylide as an Organocatalyst for Cyanosilylation Reactions Using TMSCN. ACS Appl. Mater. Interfaces 2020, 85. https://doi.org/10.1021/acs.joc.9b03347.

(13) Zhang, Z.; Chen, J.; Bao, Z.; Chang, G.; Xing, H.; Ren, Q. Insight into the Catalytic Properties and Applications of Metal-Organic Frameworks in the Cyanosilylation of Aldehydes. RSC Adv. 2015, 5 (97), 79355-79360. https://doi.org/10.1039/c5ra13102b.

(14) Sharma, M. K.; Singh, D.; Mahawar, P.; Yadav, R.; Nagendran, S. Catalytic Cyanosilylation Using Germylene Stabilized Platinum(Ii) Dicyanide. Dalt. Trans. 2018, 47 (17), 5943-5947. https://doi.org/10.1039/c8dt00043c.

(15) Wang, W.; Luo, M.; Li, J.; Pullarkat, S. A.; Ma, M. Low-Valent Magnesium(i)-Catalyzed Cyanosilylation of Ketones. Chem. Commun. 2018, 54 (24), 3042-3044. https://doi.org/10.1039/c8cc00826d. 
(16) Harinath, A.; Bhattacharjee, J.; Nayek, H. P.; Panda, T. K. Alkali Metal Complexes as Efficient Catalysts for Hydroboration and Cyanosilylation of Carbonyl Compounds. Dalt. Trans. 2018, 47 (36), 12613-12622. https://doi.org/10.1039/c8dt02032a.

(17) Sakai, T.; Miyata, K.; Tsuboi, S.; Takeda, A.; Utaka, M.; Torii, S. A Convenient Reductive Removal of Benzylic Hydroxyl and Trimethylsilyloxyl Groups with Me3SiCl-NaI-MeCN Reagent. Bull. Chem. Soc. Jpn. 1989, 62 (11), 3537-3541. https://doi.org/10.1246/bcsj.62.3537.

(18) Strappaveccia, G.; Lanari, D.; Gelman, D.; Pizzo, F.; Rosati, O.; Curini, M.; Vaccaro, L. Efficient Synthesis of Cyanohydrin Trimethylsilyl Ethers via 1,2-Chemoselective Cyanosilylation of Carbonyls. Green Chem. 2013, 15 (1), 199-204. https://doi.org/10.1039/c2gc36442e.

(19) Garnes-Portolés, F.; Rivero-Crespo, M. Á.; Leyva-Pérez, A. Nanoceria as a Recyclable Catalyst/Support for the Cyanosilylation of Ketones and Alcohol Oxidation in Cascade. $J$. Catal. 2020, 392, 21-28. https://doi.org/10.1016/j.jcat.2020.09.032.

(20) Song, J. J.; Gallou, F.; Reeves, J. T.; Tan, Z.; Yee, N. K.; Senanayake, C. H. Activation of TMSCN by N-Heterocyclic Carbenes for Facile Cyanosilylation of Carbonyl Compounds. J. Org. Chem. 2006, 71 (3), 1273-1276. https://doi.org/10.1021/jo052206u.

(21) Kolocouris, A.; Koch, A.; Kleinpeter, E.; Stylianakis, I. 2-Substituted and 2,2Disubstituted Adamantane Derivatives as Models for Studying Substituent Chemical

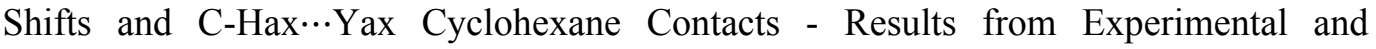
Theoretical NMR Spectroscopic Chemical Shifts and DFT Structures. Tetrahedron 2015, 71 (16), 2463-2481. https://doi.org/10.1016/j.tet.2015.01.044.

(22) Jia, Y.; Zhao, S.; Song, Y. F. The Application of Spontaneous Flocculation for the Preparation of Lanthanide-Containing Polyoxometalates Intercalated Layered Double Hydroxides: Highly Efficient Heterogeneous Catalysts for Cyanosilylation. Appl. Catal. A Gen. 2014, 487, 172-180. https://doi.org/10.1016/j.apcata.2014.09.005.

(23) Batista, P. K.; Alves, D. J. M.; Rodrigues, M. O.; De Sá, G. F.; Junior, S. A.; Vale, J. A. Tuning the Catalytic Activity of Lanthanide-Organic Framework for the Cyanosilylation of Aldehydes. J. Mol. Catal. A Chem. 2013, 379, 68-71. https://doi.org/10.1016/j.molcata.2013.07.016.

(24) Dvries, R. F.; De La Peña-Oshea, V. A.; Snejko, N.; Iglesias, M.; Gutiérrez-Puebla, E.; Monge, M. Á. Insight into the Correlation between Net Topology and Ligand Coordination Mode in New Lanthanide MOFs Heterogeneous Catalysts: A Theoretical and Experimental Approach. Cryst. Growth Des. 2012, 12 (11), 5535-5545. https://doi.org/10.1021/cg301096d.

(25) Dvries, R. F.; Iglesias, M.; Snejko, N.; Gutiérrez-Puebla, E.; Monge, M. A. Lanthanide Metal-Organic Frameworks: Searching for Efficient Solvent-Free Catalysts. Inorg. Chem. 2012, 51 (21), 11349-11355. https://doi.org/10.1021/ic300816r.

(26) Dvries, R. F.; Snejko, N.; Iglesias, M.; Gutiérrez-Puebla, E.; Monge, M. A. Ln-MOF Pseudo-Merohedral Twinned Crystalline Family as Solvent-Free Heterogeneous Catalysts. Cryst. Growth Des. 2014, $14 \quad$ (5), 2516-2521. https://doi.org/10.1021/cg5002336.

(27) Gómez, G. E.; Kaczmarek, A. M.; Van Deun, R.; Brusau, E. V.; Narda, G. E.; Vega, D.; Iglesias, M.; Gutiérrez-Puebla, E.; Monge, M. Á. Photoluminescence, UnconventionalRange Temperature Sensing, and Efficient Catalytic Activities of Lanthanide MetalOrganic Frameworks. Eur. J. Inorg. Chem. 2016, 1577-1588. 
https://doi.org/10.1002/ejic.201501402.

(28) Gómez, G. E.; Brusau, E. V.; Sacanell, J.; Soler Illia, G. J. A. A.; Narda, G. E. Insight into the Metal Content-Structure-Property Relationship in Lanthanide Metal-Organic Frameworks: Optical Studies, Magnetism, and Catalytic Performance. Eur. J. Inorg. Chem. 2018, 2452-2460. https://doi.org/10.1002/ejic.201701474.

(29) Gustafsson, M.; Bartoszewicz, A.; Martiín-Matute, B.; Sun, J.; Grins, J.; Zhao, T.; Li, Z.; Zhu, G.; Zou, X. A Family of Highly Stable Lanthanide Metal-Organic Frameworks: Structural Evolution and Catalytic Activity. Chem. Mater. 2010, 22 (11), 3316-3322. https://doi.org/10.1021/cm100503q.

(30) He, H.; Ma, H.; Sun, D.; Zhang, L.; Wang, R.; Sun, D. Porous Lanthanide-Organic Frameworks: Control over Interpenetration, Gas Adsorption, and Catalyst Properties. Cryst. Growth Des. 2013, 13 (7), 3154-3161. https://doi.org/10.1021/cg400531j.

(31) Karmakar, A.; Rúbio, G. M. D. M.; Paul, A.; Guedes da Silva, M. F. C.; Mahmudov, K. T.; Guseinov, F. I.; Carabineiro, S. A. C.; Pombeiro, A. J. L. Lanthanide Metal Organic Frameworks Based on Dicarboxyl-Functionalized Arylhydrazone of Barbituric Acid: Syntheses, Structures, Luminescence and Catalytic Cyanosilylation of Aldehydes. Dalt. Trans. 2017, 46 (26), 8649-8657. https://doi.org/10.1039/c7dt01056g.

(32) Wu, P.; Wang, J.; Li, Y.; He, C.; Xie, Z.; Duan, C. Luminescent Sensing and Catalytic Performances of a Multifunctional Lanthanide-Organic Framework Comprising a Triphenylamine Moiety. Adv. Funct. Mater. 2011, 21 (14), 2788-2794. https://doi.org/10.1002/adfm.201100115.

(33) Evans, O. R.; Ngo, H. L.; Lin, W. Chiral Porous Solids Based on Lamellar Lanthanide Phosphonates. J. Am. Chem. Soc. 2001, 123 (42), 10395-10396. https://doi.org/10.1021/ja0163772.

(34) Wang, X.; Zhang, L.; Yang, J.; Liu, F.; Dai, F.; Wang, R.; Sun, D. Lanthanide MetalOrganic Frameworks Containing a Novel Flexible Ligand for Luminescence Sensing of Small Organic Molecules and Selective Adsorption. J. Mater. Chem. A 2015, 3 (24), 12777-12785. https://doi.org/10.1039/c5ta00061k.

(35) Liu, X.; Lin, H.; Xiao, Z.; Fan, W.; Huang, A.; Wang, R.; Zhang, L.; Sun, D. Multifunctional Lanthanide-Organic Frameworks for Fluorescent Sensing, Gas Separation and Catalysis. 2016, 45, 3743-3749. https://doi.org/10.1039/c5dt04339e.

(36) An, H.; Wang, L.; Hu, Y.; Fei, F. Temperature-Induced Racemic Compounds and Chiral Conglomerates Based on Polyoxometalates and Lanthanides: Syntheses, Structures and Catalytic Properties. CrystEngComm 2015, 17 (7), 1531-1540. https://doi.org/10.1039/c4ce01802h.

(37) Fei, F.; An, H.; Meng, C.; Wang, L.; Wang, H. Lanthanide-Supported MolybdenumVanadium Oxide Clusters: Syntheses, Structures and Catalytic Properties. 2015, 1879618805. https://doi.org/10.1039/c4ra16237d.

(38) Echenique-Errandonea, E.; Pérez, J. M.; Rojas, S.; Cepeda, J.; Seco, J. M.; Fernández, I.; Rodríguez-Diéguez, A. A Novel Yttrium-Based Metal-Organic Framework for the Efficient Solvent-Free Catalytic Synthesis of Cyanohydrin Silyl Ethers. Dalt. Trans. 2021, 50 (34), 11720-11724. https://doi.org/10.1039/D1DT01953H. 